\title{
RIBBONS AND THEIR CANONICAL EMBEDDINGS
}

\author{
DAVE BAYER AND DAVID EISENBUD
}

\begin{abstract}
ABSTRACr. We study double structures on the projective line and on certain other varieties, with a view to having a nice family of degenerations of curves and $\mathrm{K} 3$ surfaces of given genus and Clifford index. Our main interest is in the canonical embeddings of these objects, with a view toward Green's Conjecture on the free resolutions of canonical curves. We give the canonical embeddings explicitly, and exhibit an approach to determining a minimal free resolution.
\end{abstract}

\section{INTRODUCTION}

What is the limit of the canonical model of a smooth curve as the curve degenerates to a hyperelliptic curve? "A ribbon" - more precisely "a ribbon on $\mathbf{P}^{1} "$ - may be defined as the answer to this riddle. A ribbon on $\mathbf{P}^{1}$ is a double structure on the projective line. Such ribbons represent a little-studied degeneration of smooth curves that shows promise especially for dealing with questions about the Clifford indices of curves.

The theory of ribbons is in some respects remarkably close to that of smooth curves, but ribbons are much easier to construct and work with. In this paper we discuss the classification of ribbons and their maps. In particular, we construct the "holomorphic differentials" - sections of the canonical bundle - of a ribbon, and study properties of the canonical embedding. Aside from the genus, the main invariant of a ribbon is a number we call the "Clifford index", although the definition for it that we give is completely different from the definition for smooth curves. This name is partially justified here, and but much more so by two subsequent works: In the paper of Fong [1993] a strong smoothing result for ribbons is proved. In the paper of Eisenbud-Green [1995] it is shown that the Clifford index of a ribbon may be re-expressed in terms of a certain notion of generalized linear series, and the semicontinuity of the Clifford index as a smooth curve degenerates to a ribbon is established. Together, these results imply that any ribbon may be deformed to a smooth curve of the same Clifford index.

Our original motivation for studying ribbons came from an attack on a conjecture of Mark Green concerning the free resolution of a canonical curve. Be-

Received by the editors March 15, 1994 and, in revised form, April 18, 1994.

1991 Mathematics Subject Classification. Primary 14H45; Secondary 14H10, 14Q05.

Key words and phrases. Ribbon, double structure, hyperelliptic curve, Clifford index, Green's conjecture, free resolution, canonical curve, $\mathrm{K} 3$ surface, $\mathrm{K} 3$ carpet.

Both authors are grateful to the NSF for partial support during the preparation of this paper. 
fore stating the conjecture, we introduce two notions. If

$$
I \subset S=k\left[x_{0}, \ldots, x_{g-1}\right]
$$

is the homogeneous ideal of a canonically embedded curve $C$ of arithmetic genus $g$, then the free resolution of $S / I$ is known to have the form

$$
\begin{aligned}
0 \rightarrow S(-g-1) & \rightarrow S^{a_{g-3}}(-g+2) \oplus S^{b_{g-3}}(-g+1) \rightarrow \\
\ldots \rightarrow & \rightarrow S^{a_{i}}(-i-1) \oplus S^{b_{i}}(-i-2) \rightarrow \ldots \\
& \rightarrow S^{a_{1}}(-2) \oplus S^{b_{1}}(-3) \rightarrow S \rightarrow S / I \rightarrow 0
\end{aligned}
$$

with $a_{g-2-i}=b_{i}$ for all $i$. In this situation the free modules notated $S^{a_{i}}(-i-1)$ above form a subcomplex, which we think of as the "two-linear part" of the resolution, since it begins with the quadrics in the ideal of the canonical curve and continues with matrices of linear forms. Because of Green's conjecture, which we are about to state, we will define the RESOLUTION CLIFFORD INDEX of $C$ to be the length of the 2-linear part of this resolution - that is, the largest $i$ for which $a_{i} \neq 0$.

By contrast, the usual CLIFFORD INDEX of a smooth curve $C$ of genus $g \geq 3$ is defined as the maximum, over all line bundles $L$ on $C$ such that $\mathrm{h}^{0}(L)>1$ and $\mathrm{h}^{1}(L)>1$ of the quantity

$$
\text { Cliff } \begin{aligned}
L & \stackrel{\text { def }}{=} \text { degree } L-2\left(\mathrm{~h}^{0}(L)-1\right) \\
& =g+1-\mathrm{h}^{0}(L)-\mathrm{h}^{1}(L) .
\end{aligned}
$$

With this terminology, GREen's CONJECTURE on CANONICAL CURVES is the assertion that the Clifford index and the resolution Clifford index agree for smooth curves over an algebraically closed field of characteristic 0 . In terms of the new Clifford index we define for a ribbon, we make the

Canonical Ribbon Conjecture. The resolution Clifford index and the Clifford index agree for ribbons over a field of characteristic 0 .

Because of the smoothing results of Fong [1993] and Eisenbud-Green [1995, Proposition 2.3], a proof of our conjecture for some ribbon of each genus and Clifford index would imply Green's conjecture for a generic curve of each Clifford index (that is, for a generic curve in some component of the locus of curves of each Clifford index). The restriction to characteristic 0 is really necessary in both cases, since examples of Schreyer for smooth curves and examples given below for ribbons show that the conjectures sometimes fail in finite characteristic.

Perhaps the most important difference between the case of ribbons and in the case of smooth curves is that two smooth curves of the same Clifford index and genus may have different graded betti numbers, but the graded betti numbers of a ribbon are completely determined by the genus and Clifford index. This is because all ribbons of given genus and Clifford index are hyperplane sections of a particular "K3 carpet" - a double structure on a 2-dimensional rational normal scroll (at least over an algebraically closed field of characteristic 0 , this is the unique double structure on the scroll with trivial canonical bundle that 
can be embedded in the ambient space of the usual embedding of the scroll see Hulek and Van de Ven [1985]). K3 carpets are the subject of a planned paper by Eisenbud and Schreyer.

Aside from this, what we know about the canonical ribbon conjecture is rather similar to what we know of Green's conjecture itself. We can prove the analogues of Noether's and Petri's Theorems, which deal with the cases of Clifford index 1 and 2. We can also prove, by machine computation, that the conjecture is true in all cases up to genus 12 (see the table of results at the end of section 8). The proofs of these special cases are quite different in the case of ribbons, and are in a sense more direct and algebraic, than in the case of smooth curves, so that we are hopeful that the study of ribbons will be useful in further work on Green's conjecture.

We next discuss the material of this paper in more detail: The first section below is devoted to the general theory of ribbons. Here we work with double structures on a more general reduced scheme $D$. First we classify the ribbons on $D$ by certain extensions of the sheaf of differentials of $D$ (this familiar idea goes back at least to Lichtenbaum-Schlessinger [1967]). Next we describe morphisms: given a morphism from $D$ to another scheme $X$, we explain what data is necessary to describe morphisms from the ribbon to $X$. From this description we show how to tell when the extended morphism is a closed immersion. In case $X$ is a projective space, we further explain how to tell whether the image of the ribbon is "arithmetically of depth $\geq 2$ ", the analogue of the condition "projectively normal" for smooth varieties. Finally we show that the morphisms from the ribbon to another ribbon on $D$ which induce the identity on $D$ are precisely those obtained by blowing up closed subschemes of $D$.

For most of the rest of the paper we specialize to the case where the underlying scheme $D$ is the projective line over an algebraically closed field $k$ (although many of our results could be generalized at least to the case where $D$ is a nonsingular curve over an arbitrary field). We will call such a ribbon a RATIONAL RIBBON, or simply a ribbon when the context is clear.

In section 2 we specialize the classification theory to rational ribbons. Here the two fundamental invariants are the (arithmetic) genus and the Clifford index. The latter is defined in terms of the restricted cotangent sequence. An alternate, and perhaps the quickest definition is the following: We say that a ribbon is split, or "hyperelliptic" if it is isomorphic to $\mathbf{P}^{1} \times \operatorname{Spec} k[\epsilon] /\left(\epsilon^{2}\right)$ and we define the Clifford index of a ribbon $C$ as the minimum number of blowups of $C$ at reduced points of $C_{\text {red }}$ necessary to obtain a split ribbon. Some preliminary evidence is given that this notion of Clifford index is the "right" one, in the sense that it parallels the properties of the Clifford index for smooth curves. For example it is shown that a ribbon of Clifford index $a$ has a "generalized linear series" of dimension 1 and degree $a+2$ in a suitable sense. Much further justification may be found in the rest of the paper. Thus the theory of ribbons gives one direct access to curves of arbitrary genus and Clifford index.

In section 3 we present a different view of the construction and classification of ribbons, this time by gluing together ribbons on the affine line (such a ribbon is necessarily of the form Spec $k[s, \epsilon] /(\epsilon)^{2}$.) We give the translation between the necessary "gluing data" and the data of the restricted cotangent sequence 
of the ribbon. This version of the classification, though somewhat computational, is necessary for our treatment of line bundles, and in particular for our computation of the canonical embedding of the ribbon.

In section 4 we discuss line bundles and their global sections on a ribbon. The Picard group of line bundles on a ribbon of genus $g$ is simply $k^{g} \times \mathbf{Z}$, where the second factor is given by the degree of the restriction of the line bundle to $\mathbf{P}^{1}$ (equal to half the degree of the line bundle itself). The line bundles of degree 0 form a formally principle homogeneous space under the group $\mathrm{H}^{1}\left(\mathscr{O}_{\mathbf{P}^{1}}(-g-1)\right) \cong k^{g}$, and this accounts for the first factor. The global sections of line bundles are computed in terms of an exact sequence coming from restriction to $\mathbf{P}^{1}$.

After these preliminaries, we turn to the main concern of the paper, the canonical embedding of a ribbon. The canonical bundle is discussed in section 5 , using the theory developed in sections 3 and 4 to identify the global sections. We prove "Noether's Theorem for Ribbons": the canonical series provides an embedding of any nonhyperelliptic ribbon, and the canonical image is arithmetically Cohen-Macaulay (and thus Gorenstein). (One of the proofs we give of this fact involves knowing the structure of the normal bundle of the rational normal curve explicitly. This structure is folklore, but we know no reference; we provide a proof, together with the corresponding results for all the osculating bundles and their quotients, in an appendix at the end of the paper.) The canonical embedding gives a third view of the the classification of ribbons: giving a nonhyperelliptic ribbon of genus $g$ is the same as giving a line bundle contained in the normal bundle of the rational normal curve of degree $g-1$.

In section 6 we present a result obtained jointly with Joe Harris which shows that ribbons always represent smooth points on the Hilbert scheme of canonically embedded curves.

Unlike the case of smooth curves, it is possible to deal with canonical embeddings of ribbons by induction on the genus. To do this, we prove in section 7 that the image in $\mathbf{P}^{g-2}$ of the projection of a canonically embedded ribbon in $\mathbf{P}^{g-1}$ from a point on the ribbon is the canonical embedding of the ribbon obtained from $C$ by blowing up the point. This also leads to an easy proof of one inequality of the canonical ribbon conjecture: The resolution Clifford index is always $\geq$ the Clifford index.

In this section we also show that the only nonhyperelliptic ribbon that can be embedded in a smooth surface is the double conic in $\mathbf{P}^{2}$. This suggests one reason why the theory of ribbons has not been pursued so much before: The double structures on $\mathbf{P}^{1}$ that one sees most often are all split ribbons, and thus not of much interest.

We have already mentioned that all ribbons are hyperplane sections of suitable (nonreduced) K3 surfaces. In the last section of the paper we give the part of the theory of these $\mathrm{K} 3$ carpets which is most relevant to the canonical ribbon conjecture, explaining in particular how to construct a nonminimal free resolution for the homogeneous coordinate ring of a ribbon, and - conjecturally how to make it minimal. We include some numerical evidence, produced by the program Macaulay of Bayer and Stillman [1990] for our conjectures, and thus for Green's Conjecture.

A quasi-mathematical remark: Each of the people who has worked on Green's 
Conjecture probably has his/her own favorite nonmimal resolution of some degenerate curve of genus $g$, and a conjecture about minimalizing it that would imply at least the generic case of Green's conjecture. So far as we know the method of ribbons is the only one that gives such possibilities for the generic curve of each Clifford index. There are several ways of making nonmiminal resolutions of ribbons, and attempting to minimalize them. The method of $\mathrm{K} 3$ carpets, presented in section 8 , seems to us the one with the fewest choices involved.

We are grateful as always to Joe Harris for numerous helpful comments and suggestions, as well as for a number of specific results, which are attributed to him below.

Throughout this paper, we work over a fixed field $k$. By a scheme we shall mean a scheme of finite type over $k$.

\section{GENERAL RIBBONS AND THEIR MORPHISMS}

We begin with some basic definitions and remarks. Throughout this section, $D$ will denote a reduced connected scheme over the ground field $k$.

A RIBBON ON $D$ is a scheme $C$ equipped with an isomorphism $D \rightarrow C_{\text {red }}$, the reduced scheme of $C$, such that the ideal sheaf $\mathscr{L}$ of $D$ in $C$ satisfies

$$
\mathscr{L}^{2}=0 \text {. }
$$

Because of this condition, $\mathscr{L}$ may be regarded as a sheaf on $D$, and we further require that

$\mathscr{L}$ is a line bundle on $D$.

Note that the subscheme $D$ is determined by $C$ as $C_{\text {red }}$. The line bundle $\mathscr{L}$ is the Conormal BundLe of $D$ in $C$.

A RIBBON is simply a scheme $C$ which is a ribbon on $C_{\text {red }}$.

We shall say that the the ribbon $C$ is SPLIT if the inclusion $D \hookrightarrow C$ admits a retraction $C \rightarrow D$.

The following result is an elementary but important special case of the Classification Theorem below:

Proposition 1.1. Given a reduced connected scheme $D$ and a line bundle $\mathscr{L}$ on $D$ there is a unique split ribbon on $D$ with conormal bundle $\mathscr{L}$.

Proof. If we embed $D$ in the total space $X$ of $\mathscr{L}$, then the first infinitesimal neighborhood of $D$ in $X$ is a split ribbon on $D$ with conormal bundle $\mathscr{L}$.

To prove uniqueness, let $C$ be any split ribbon with conormal bundle $\mathscr{L}$. Because $C$ is split, the natural exact sequence

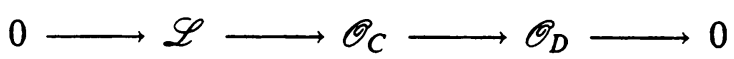

is a sequence of $\mathscr{O}_{D}$-modules. It is split as a sequence of $\mathscr{O}_{D}$-modules because the identity element of $\mathscr{O}_{C}$ lifts the identity element of $\mathscr{O}_{D}$. Because $\mathscr{L}^{2}=0$, the algebra structure of $O_{C}$ is determined by the module structure of $\mathscr{L}$.

There are also lots of ribbons that are not split-we shall see in the next section that the simplest example is given by the quartic plane curve whose equation is the square of that of a nonsingular conic in $\mathbf{P}^{2}$. To classify the nonsplit ribbons, we will say that two ribbons $C$ and $C^{\prime}$ on $D$ are IsomorpHIC OVER $D$ if there is an isomorphism between them that extends the identity on 
$D$. More generally, a morphism $C \rightarrow C^{\prime}$ over $D$ is by definition a morphism extending the identity morphism $D \rightarrow D$.

Given a ribbon $C$ on $D$ with conormal bundle $\mathscr{L}$ we define the RESTRICTED COTANGENT SEQUENCE of $C$ to be the natural short exact sequence

$$
\left.0 \longrightarrow \mathscr{L} \longrightarrow \Omega_{C}\right|_{D} \longrightarrow \Omega_{D} \longrightarrow 0 .
$$

The restricted cotangent sequence defines the EXTENSION CLASS

$$
e_{C} \in \operatorname{Ext}_{D}^{1}\left(\Omega_{D}, \mathscr{L}\right)
$$

of $C$.

The following classification is closely related to the ideas of Lichtenbaum and Schlessinger [1967]:

Theorem 1.2 (Classification Theorem). Given any line bundle $\mathscr{L}$ on a reduced connected scheme $D$, and any class

$$
e \in \operatorname{Ext}_{D}^{1}\left(\Omega_{D}, \mathscr{L}\right)
$$

there is a unique ribbon $C$ on $D$ with $e=e_{C}$.

If $D$ is proper over $k$ and $e^{\prime} \in \operatorname{Ext}_{D}^{1}\left(\Omega_{D}, \mathscr{L}\right)$ is another class, corresponding to a ribbon $C^{\prime}$, then $C \cong C^{\prime}$ iff $e=a e^{\prime}$ for some $a \in k^{*}$.

Proof. Let $d: \mathscr{O}_{D} \rightarrow \Omega_{D}$ be the canonical derivation, and consider an extension

$$
e: 0 \longrightarrow \mathscr{L} \longrightarrow \mathscr{E} \stackrel{\phi}{\longrightarrow} \Omega_{D} \longrightarrow 0 .
$$

Define $\mathscr{O}_{C}$ as a sheaf of abelian groups to be the pullback

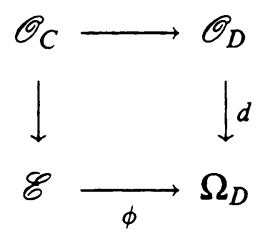

so that we have the commutative diagram

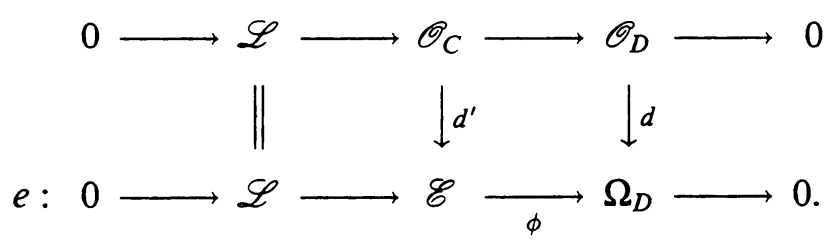

We make $\mathscr{O}_{C}$ into a sheaf of $k$-algebras as follows: if $a_{1}, a_{2}$ are sections of $\mathscr{O}_{D}$ over an open set $U$ of $D$, and $x_{1}, x_{2}$ are sections of $\mathscr{E}$ over $U$ with

$$
d a_{i}=\phi x_{i}
$$

so that each $\left(a_{i}, x_{i}\right)$ is a section of $\mathscr{O}_{C}$ on $U$, we define

$$
\left(a_{1}, x_{1}\right)\left(a_{2}, x_{2}\right)=\left(a_{1} a_{2}, a_{1} x_{2}+a_{2} x_{1}\right),
$$

the last being again a section of $\mathscr{O}_{C}$ because $d$ is a derivation. We may now define

$$
C=\operatorname{Spec} \mathscr{O}_{C}
$$


and check that it is a ribbon on $D$. It is also easy to check that

$$
d^{\prime}: \mathscr{O}_{C} \rightarrow \mathscr{E}
$$

is the universal $k$-linear derivation of $\mathscr{O}_{C}$ to an $\mathscr{O}_{D}$-module. Thus $d^{\prime}$ is the restriction of the universal $k$-linear derivation, and

$$
\left.\mathscr{E} \cong \Omega_{C}\right|_{D}
$$

is the restriction of the module of differentials $\Omega_{C}$. This shows that $C$ is a ribbon with extension class $e$, proving the first part of the Theorem.

To prove the second part, note first that if $C$ and $C^{\prime}$ are ribbons over $D$ then a morphism $f: C \rightarrow C^{\prime}$ over $D$ is a map $f^{*}: \mathscr{O}_{C^{\prime}} \rightarrow \mathscr{O}_{C}$ of sheaves of $k$-algebras inducing the identity on $\mathscr{O}_{D}$. Such a map induces a map of the restricted cotangent sequences

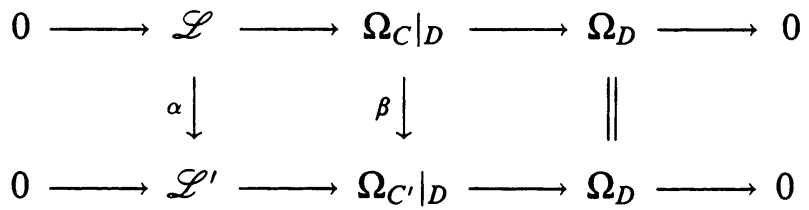

where $\beta$ is the map induced by $d f^{*}$ and $\alpha$ is the map induced by $\beta$. If $f$ is an isomorphism, then the $\beta$ is an isomorphism and it follows that $\alpha$ is too. Since $D$ is reduced, connected, and proper over $k$, and $\mathscr{L}$ is a line bundle, the only automorphisms of $\mathscr{L}$ are the elements of $k^{*}$, so $\alpha \in k^{*}$. The given map of exact sequences corresponds to the map induced by $\alpha$ on $\operatorname{Ext}_{D}^{1}\left(\Omega_{D}, \mathscr{L}\right)$, so that $e$ and $e^{\prime}$ differ by an element of $k^{*}$ as required.

Conversely, any element of $k^{*}$, regarded as a map

$$
\alpha: \mathscr{L} \rightarrow \mathscr{L}^{\prime},
$$

induces an isomorphism of exact sequences as above. Using the constructions of $C$ and $C^{\prime}$ from their restricted cotangent sequences, we may reverse the process and see that if $e$ and $e^{\prime}$ differ by an element of $k^{*}$ then $C \cong C^{\prime}$.

Corollary 1.3. If $D$ is a smooth affine variety over $k$, then every ribbon on $D$ is split.

Proof. In this case $\Omega_{D}$ is a projective $\mathscr{O}_{D}$-module, so

$$
\operatorname{Ext}_{D}^{1}\left(\Omega_{D}, \mathscr{L}\right)=0 .
$$

Corollary 1.4. If $D$ is reduced, connected, and proper over $k$, then the set of nonsplit ribbons on $D$ with conormal bundle $\mathscr{L}$, up to isomorphism over $D$, is in one-to-one correspondence with the points of the projective space of lines in $\operatorname{Ext}_{D}^{1}\left(\Omega_{D}, \mathscr{L}\right)$.

So far our classification has been up to "isomorphism over $D$ ", that is, up to isomorphisms inducing the identity on $D$. It is easy to turn this into a classification up to abstract isomorphism:

Corollary 1.5. If $D$ is reduced, connected, and proper over $k$, then the set of isomorphism classes of nonsplit ribbons $C$ such that $C_{\text {red }} \cong D$, and such that the conormal bundle of $C_{\mathrm{red}}$ is isomorphic to a given line bundle $\mathscr{L}$ on $C_{\mathrm{red}}$, is the 
projective space of lines in $\operatorname{Ext}_{D}^{1}\left(\Omega_{D}, \mathscr{L}\right)$ modulo the group of automorphisms of $D$ preserving $\mathscr{L}$.

Proof. Given a ribbon $C$ as in the Corollary, two choices of the structure of a ribbon on $D$ differ by a unique element of Aut $D$.

The morphisms from a ribbon $C$ to a scheme $X$ admit a simple description in terms of the induced map $f: D=C_{\text {red }} \rightarrow X$. Given such a map $f$, we write $d f: f^{*} \Omega_{X} \rightarrow \Omega_{D}$ for the induced map on the sheaves of differentials, and we write

$$
d f^{*}: \operatorname{Ext}_{D}^{1}\left(\Omega_{D}, \mathscr{L}\right) \longrightarrow \operatorname{Ext}_{D}^{1}\left(f^{*} \Omega_{X}, \mathscr{L}\right)
$$

for the map induced by $d f$ on Ext. Note that if $C$ is a ribbon, and $f: D \rightarrow C$ is the inclusion, then $f^{*} \Omega_{X}=\left.\Omega_{C}\right|_{D}$. We shall often write $\left.\Omega_{X}\right|_{D}$ for $f^{*} \Omega_{X}$ also in the general case.

Theorem 1.6. If $C$ is a ribbon on $D$ with restricted cotangent sequence

$$
\left.0 \longrightarrow \mathscr{L} \rightarrow \Omega_{C}\right|_{D} \underset{\phi}{\longrightarrow} \Omega_{D} \longrightarrow 0,
$$

then the morphisms from $C$ to a scheme $X$ extending a given morphism $f$ : $D \rightarrow X$ are in one-to-one correspondence with the splittings of the exact sequence $d f^{*} e_{C}$; that is, with the maps of sheaves

$$
g:\left.\left.\Omega_{X}\right|_{D} \rightarrow \Omega_{C}\right|_{D}
$$

making the diagram

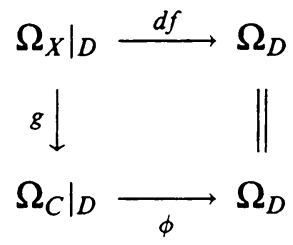

commutative.

In particular a morphism extending $f$ exists iff

$$
d f^{*} e_{C}=0 .
$$

Proof. Since $\mathscr{O}_{C}$ is a sheaf of $k$-algebras on $D, f_{*} \mathscr{O}_{C}$ is a sheaf of $k$-algebras on $X$, and the morphisms $\tilde{f}: C \rightarrow X$ extending $f$ are in one-to-one correspondence with the maps of algebras $\tilde{f}^{\#}: \mathscr{O}_{X} \rightarrow f_{*} \mathscr{O}_{C}$ lifting the map $f^{\#}$ : $\mathscr{O}_{X} \rightarrow f_{*} \mathscr{O}_{D}$.

Since $f_{*}$ is left exact, $f_{*} \mathscr{O}_{C}$ is the pullback of the diagram

$$
\left.f_{*} \Omega_{C}\right|_{f_{*} \phi} f_{*} \Omega_{\mathcal{O}_{D}}
$$

Thus the desired algebra maps are in one-to-one correspondence with the derivations of sheaves $\left.\mathscr{O}_{X} \rightarrow f_{*} \Omega_{C}\right|_{D}$ lifting the derivation

$$
\mathscr{O}_{X} \stackrel{f^{*}}{\longrightarrow} f_{*} \mathscr{O}_{D} \rightarrow f_{*} \Omega_{D}
$$


Such derivations correspond uniquely to maps of sheaves of modules

$$
g^{\prime}:\left.\Omega_{X} \rightarrow f_{*} \Omega_{C}\right|_{D}
$$

making the diagram

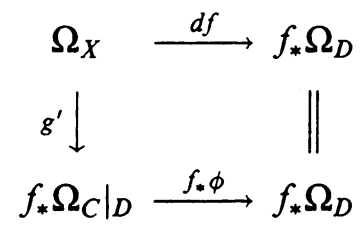

commute. By the adjointness of $f_{*}$ and $f^{*}$, such $g^{\prime}$ are in one-to-one correspondence with the maps $g$ described in the theorem.

Corollary 1.7. If $C$ is a ribbon on $D$ with restricted cotangent sequence $e_{C}$, then the maps $f: C \rightarrow D$ such that the composite

$$
D \hookrightarrow C \stackrel{f}{\longrightarrow} D
$$

is the identity are in one-to-one correspondence with the splittings of $e_{C}$.

The following gives a useful criterion for a map from a ribbon to be a closed immersion. Given a closed immersion into projective space, it also tells us when the homogeneous coordinate ring of the image has depth $\geq 2$, which is the analogue for ribbons of projective normality for smooth varieties.

If $f: C \rightarrow X$ is a morphism such that $\left.f\right|_{D}$ is a closed immersion, then we shall write $N_{D, f}^{\prime}$ for the pullback to $D$ of the conormal sheaf of $f(D)$ in $X$; that is,

$$
N_{D, f}^{\prime} \stackrel{\text { def }}{=} f^{*}\left(\mathscr{I}_{f(D) / X} / \mathscr{I}_{f(D) / X}^{2}\right),
$$

where $\mathscr{I}_{f(D) / X}$ is the ideal sheaf of $f(D)$ in $X$. In this situation we define $\alpha_{f}: N_{D, f}^{\prime} \rightarrow \mathscr{L}$ to be the pullback of the quotient map $\mathscr{I}_{f(D) / X} \rightarrow \mathscr{I}_{f(C) / X}$.

Theorem 1.8. Let $C$ be a ribbon on $D$ with conormal bundle $\mathscr{L}$.

(1) If

$$
f: C \rightarrow X
$$

is a morphism, then $f$ is a closed immersion iff the restriction of $f$ to $D$ is a closed immersion and $\alpha_{f}$ is an epimorphism.

(2) If

$$
f: C \rightarrow \mathbf{P}^{r}
$$

is a closed immersion, and the homogeneous coordinate ring of $f(D)$ has depth $\geq 2$, then the homogeneous coordinate ring of $f(C)$ has depth $\geq 2$ iff the map

$$
\alpha_{f *}(n): \mathrm{H}^{0}\left(N_{D, f}^{\prime}(n)\right) \rightarrow \mathrm{H}^{0}(\mathscr{L}(n))
$$

induced by $\alpha_{f}$ is surjective for all integers $n$.

Proof. (1) $f$ is a closed immersion iff the induced map $f^{*}: \mathscr{O}_{X} \rightarrow f_{*} \mathscr{O}_{C}$ is an epimorphism, and similarly for the restriction of $f$ to $D$. Since $D$ is a subscheme of $C$, it is clear that $f$ can be a closed immersion on $C$ only if it 
is a closed immersion on $D$. Now suppose that $f$ is a closed immersion on $D$ and consider the diagram

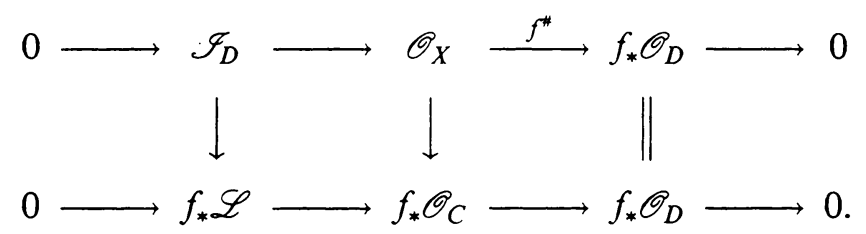

From the snake lemma we see that the middle vertical map is an epimorphism iff the left-hand vertical map is. Since $f_{*} \mathscr{L}$ is supported on $f(D) \cong D$, this map is an epimorphism iff $\alpha_{f}$ is, proving part (1).

(2) The homogeneous coordinate ring of $f(C)$ has depth $\geq 2$ iff the map

$$
f_{*}(n): \mathrm{H}^{0}\left(\mathscr{O}_{\mathbf{P r}}(n)\right) \rightarrow \mathrm{H}^{0}\left(\mathscr{O}_{C}(n)\right)
$$

induced by $f$ is surjective for all integers $n$. Note that, because of our hypothesis on $D$, the maps

$$
f_{*}(n): \mathrm{H}^{0}\left(\mathscr{O}_{\mathbf{P r}}(n)\right) \rightarrow \mathrm{H}^{0}\left(\mathscr{O}_{D}(n)\right)
$$

are all surjective. Thus setting $X=\mathbf{P}^{r}$ in the above diagram, twisting by $\mathcal{O}_{\mathrm{Pr}}(n)$, and taking $\mathrm{H}^{0}$, the desired result follows again from the snake lemma.

The maps between ribbons on $D$ which induce the identity map on $D$ have a particularly nice description: they are just the blow-ups of Cartier divisors on $D$ (these are Weil divisors on $C$ ). First we analyze such blow-ups.

Let $\delta \subset D$ be an effective Cartier divisor in $D$, and let $C$ be a ribbon on $D$ with conormal bundle $\mathscr{L}$ and extension class $e_{C}$. Let $\alpha: \mathscr{L} \rightarrow \mathscr{L}(\delta)$ be multiplication by a section of $\mathscr{O}_{D}(\delta)$ corresponding to $\delta$, and let $C^{\prime}$ be the ribbon corresponding to the extension class

$$
e_{C^{\prime}}=\alpha\left(e_{C}\right) \text {, }
$$

where we have written $\alpha$ again for the induced map

$$
\operatorname{Ext}_{D}^{1}\left(\Omega_{D}, \mathscr{L}\right) \rightarrow \operatorname{Ext}_{D}^{1}\left(\Omega_{D}, \mathscr{L}^{\prime}\right) .
$$

With this notation we have:

Theorem 1.9. If $X \rightarrow C$ is the blow-up of $C$ along $\delta$, and $C^{\prime}$ is the ribbon corresponding to $\alpha\left(e_{C}\right)$ as above, then $X \cong C^{\prime}$, and the blow-up map corresponds to the map of exact sequences

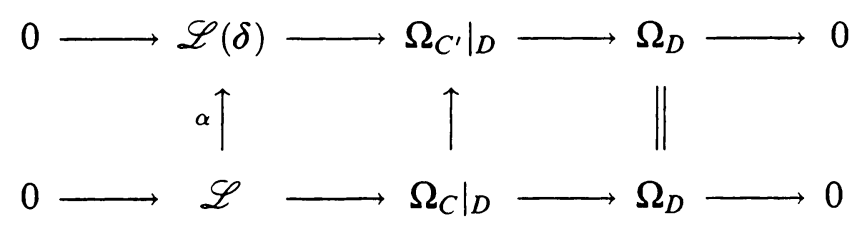

induced by $\alpha$.

Proof. The matter is local on $C$, so we may assume that $C=\operatorname{Spec} A$, that $\mathscr{L}$ is the trivial bundle, so that the nilpotent ideal of $A$ is generated by one element $y$, and that the ideal of $\delta$ in $D$ is principal. Let $x \in A$ be any element of $A$ lifting the generator of the ideal of $\delta$ in $\mathscr{O}_{D}(D)=A /(y)$. Since $\delta$ is Cartier, 
$x$ is a nonzerodivisor on $A /(y)$ and thus on $A$. Of course $y^{2}=0$. With these choices it is easy to check that

$$
C^{\prime}=\operatorname{Spec} A[y / x],
$$

where $A[y / x]$ is the subring generated by $y / x$ in the localization $A\left[x^{-1}\right]$.

Consider now the blow-up. Let $B=A \oplus(x, y) \oplus(x, y)^{2} \oplus \ldots$, so that $X=$ $\operatorname{Proj}(B)$. Since $y$ is nilpotent $X$ is affine, and we have $X \cong \operatorname{Spec}\left(B\left[x^{-1}\right]_{0}\right)$. Since $(x, y)^{n}=x^{n-1}(x, y)$, we get $B\left[x^{-1}\right]_{0}=A[y / x]$ as required.

It follows that we can describe all maps of ribbons over $D$ in these terms. For simplicity we stick to the case where $D$ is irreducible:

Corollary 1.10. Suppose that $D$ is irreducible, and let $f: C^{\prime} \rightarrow C$ be a map of ribbons over $D$. If the image of $f$ is contained in $D$, then $C^{\prime}$ is split and $f$ is the projection. Otherwise, $f$ is the blowup of $C$ along a subscheme that is a Cartier divisor in $D$.

Proof. Let $\mathscr{L}$ and $\mathscr{L}^{\prime}$ be the conormal bundles of $C$ and $C^{\prime}$. The map

$$
\left.\left.\Omega_{C}\right|_{D} \rightarrow \Omega_{C^{\prime}}\right|_{D}
$$

corresponding to $f$ induces a map $\alpha: \mathscr{L} \rightarrow \mathscr{L}^{\prime}$ as in the preceding theorem. Since $D$ is irreducible, $\alpha$ is an inclusion corresponding to some Cartier divisor $\delta$. The rest follows as in the theorem.

\section{RATIONAL RIBBONS 1: THE RESTRICTED COTANGENT SEQUENCE}

In this section we shall suppose that the field $k$ is algebraically closed, and we shall consider only ribbons $C$ on $D=\mathbf{P}^{1}=\mathbf{P}_{k}^{1}$. Here the classification described in section 1 becomes much more concrete.

As usual with curves, the fundamental invariant of $C$ is its genus, here defined as the arithmetic genus

$$
g(C)=1-\chi\left(\mathscr{O}_{C}\right)=1-\mathrm{H}^{0} \mathscr{O}_{C}+\mathrm{H}^{1} \mathscr{O}_{C} .
$$

From the additivity of $\chi$ and the fact that $\chi\left(\mathscr{O}_{\mathbf{P}^{1}}(n)\right)=n+1$ we see that $C$ has genus $\mathrm{g}$ iff the conormal bundle $\mathscr{L}$ of $\mathbf{P}^{1}=C_{\text {red }}$ in $C$ is $\mathscr{O}_{\mathbf{P}^{1}}(-g-1)$.

$C$ is split iff the the inclusion $D \hookrightarrow C$ admits a section. Such a section is a map $C \rightarrow \mathbf{P}^{1}$, which will have degree 2 in the sense that the scheme-theoretic fiber of a point in $\mathbf{P}^{1}$ has length 2 . Conversely, any degree 2 map $f: C \rightarrow \mathbf{P}^{1}$, induces a degree 1 map $f_{\text {red }}: D \hookrightarrow C \rightarrow \mathbf{P}^{1}$. Composing $\mathrm{f}$ with the inverse of this isomorphism of $\mathbf{P}^{1}$ we obtain a section of $D \hookrightarrow C$. Because of this we will call $C$ HYPERELLIPTIC if $C$ is split.

We have used the word hyperelliptic because hyperelliptic ribbons have many properties in common with smooth hyperelliptic curves. As a first example, we note that if $g \geq 2$, then there are no nontrivial maps $\Omega_{\mathbf{P}^{1}} \rightarrow \mathscr{O}_{\mathbf{P}^{1}}(-g-1)$, so by Corollary 1.7 there is at most one splitting of $e_{C}$, and the two to one map $C \rightarrow \mathbf{P}^{1}$ is unique if it exists. On the other hand, in the cases $g=0$ and $g=1$ there are, again by Corollary 1.7, one-parameter and two-parameter families of such two-to-one maps, respectively, just as in the case of smooth curves.

From Corollaries 1.4 and 1.5 we deduce: 
Theorem 2.1. The set of nonhyperelliptic ribbons of genus $g$ on $\mathbf{P}^{1}$, up to isomorphism on $\mathbf{P}^{1}$, is the set

$$
\mathbf{P}^{g-3}=\mathbf{P}\left(\mathbf{H}^{0}\left(\mathscr{O}_{\mathbf{P}^{1}}(g-3)\right)\right),
$$

the space of 1-quotients of $\mathrm{H}^{0}\left(\mathscr{O}_{\mathbf{P}^{1}}(g-3)\right)$.

The set of abstract isomorphism classes of nonhyperelliptic ribbons of genus $g$ on $\mathbf{P}^{1}$ is thus this set modulo Aut $\mathbf{P}^{1}$.

Proof. By Corollary 1.4, it is enough to identify the lines in

$$
\begin{aligned}
\operatorname{Ext}_{D}^{1}\left(\Omega_{D}, \mathscr{O}_{\mathbf{P}^{1}}(-g-1)\right) & =\mathrm{H}^{1}\left(\Omega_{D}^{*} \otimes \mathscr{O}_{\mathbf{P}^{1}}(-g-1)\right) \\
& =\mathrm{H}^{1}\left(\mathscr{O}_{\mathbf{P}^{1}}(-g+1)\right),
\end{aligned}
$$

with the 1-quotients of $\mathrm{H}^{0}\left(\mathscr{O}_{\mathbf{P}^{1}}(g-3)\right)$, and this is simply Serre duality.

For ribbons on $\mathbf{P}^{1}$ we may write the restricted cotangent sequence in the simple form

$$
0 \rightarrow \mathscr{O}_{\mathbf{P}^{1}}(-g-1) \rightarrow \mathscr{O}_{\mathbf{P}^{1}}(-a-2) \oplus \mathscr{O}_{\mathbf{P}^{1}}(-b-2) \rightarrow \mathscr{O}_{\mathbf{P}^{1}}(-2) \rightarrow 0
$$

for some integers $a$ and $b$ with $0 \leq a \leq b \leq g-1$ and $a+b=g-1$. We define the CLIFFORD INDEX of $C$ to be the integer $a$. Note that the sequence and thus the ribbon is split, that is, hyperelliptic, iff $a=0$, as for a smooth curve. And just as in the case of smooth curves, the Clifford index takes values from 0 to $(g-1) / 2$.

To give a sequence of the sort above, it suffices to specify the right-hand map

$$
\mathscr{O}_{\mathbf{P}^{1}}(-a-2) \oplus \mathscr{O}_{\mathbf{P}^{1}}(-b-2) \rightarrow \mathscr{O}_{\mathbf{P}^{1}}(-2),
$$

which must be an epimorphism of sheaves. If we choose coordinates, and thus identify the homogeneous coordinate ring of $\mathbf{P}^{1}$ with the polynomial ring in 2 variables $S=k[s, t]$, then such a map is given by a pair of homogeneous polynomials $\alpha$ and $\beta$ of degrees $a$ and $b$ respectively. The condition that the map be an epimorphism is then simply the condition that $\alpha, \beta$ is a regular sequence, and the restricted cotangent sequence itself is the Koszul complex of $\alpha, \beta$ twisted by -2 . We shall write $I_{C}=(\alpha, \beta)$ for the ideal associated in this way to the ribbon $C$. It is easy to see that $I_{C}$ is an invariant of the sequence $e_{C}$ and thus of $C$.

To make a connection with Theorem 2.1 we recall a result that seems to have been discovered by Macaulay. To express it, we write $S_{d}$ for $\mathrm{H}^{0}\left(\mathscr{O}_{\mathbf{P}^{1}}(d)\right)$, the vectorspace of homogeneous forms of degree $d$.

Theorem 2.2. There is a one-to-one correspondence between hyperplanes in $S_{g-3}$ and ideals generated by regular sequences $(\alpha, \beta) \subset S$ whose generators have degrees $a, b$ with $a+b=g-1$, given as follows: If $I=(\alpha, \beta) \subset S$ is such an ideal, then the subspace

$$
H(I)=(\alpha, \beta) \cap S_{g-3}
$$

is a hyperplane; and if $H \subset S$ is a hyperplane, then the ideal $I(H)$ consisting of all homogeneous polynomials $\gamma$ of degree $c \leq g-3$ such that

$$
\gamma(s, t)^{g-3-c} \subset H
$$


together with all forms of degrees $>g-3$ is generated by a regular sequence of elements of suitable degrees.

In the situation of the theorem, we will call any functional $\phi: S_{g-3} \rightarrow k$ with kernel $H(I)$ a DUAL SOCLE GENERATOR for $I$. The following additional information is also well-known:

Proposition 2.3. The lines in

$$
\operatorname{Ext}_{D}^{1}\left(\Omega_{D}, \mathscr{O}_{\mathbf{P}^{1}}(-g-1)\right)=\mathrm{H}^{1}\left(\Omega_{D}^{*} \otimes \mathscr{O}_{\mathbf{P}^{1}}(-g-1)\right)=\left(\mathbf{H}^{0}\left(\mathscr{O}_{\mathbf{P}^{1}}(g-3)\right)\right)^{*}
$$

spanned by the class of the Koszul complex of $(\alpha, \beta)$ and the class of a dual socle generator for $(\alpha, \beta)$ are the same.

Thus the element in $\mathbf{P}\left(\mathrm{H}^{0}\left(\mathscr{O}_{\mathbf{P}^{1}}(g-3)\right)\right)$, associated by Theorem 2.1 to a ribbon $C$ is the same as the element corresponding to a dual socle generator $f$ for $I_{C}$.

Using this, we can make the classification theorem more geometric:

Corollary 2.4. A ribbon $C$ on $\mathbf{P}^{1}$ is determined by the set of divisors $\delta \subset \mathbf{P}^{1}$ such that the blow-up of $C$ along $\delta$ is hyperelliptic.

In fact, if we write $\delta \in S$ for a polynomial defining the divisor $\delta$, then the set of divisors

$$
\{\delta \mid \text { the blow-up of } C \text { along } \delta \text { is hyperelliptic }\}
$$

is the same as the set of forms

$$
\left\{\delta \mid \delta \in I_{C}\right\}
$$

In particular, the Clifford index of $C$ is the minimal number of blow-ups of $C$ at reduced points necessary to reach a hyperelliptic ribbon.

Proof. If we write $C^{\prime}$ for the blown-up ribbon, then $e_{C^{\prime}}$ is obtained by pushing forward the sequence $e_{C}$ along the map induced by $\delta$,

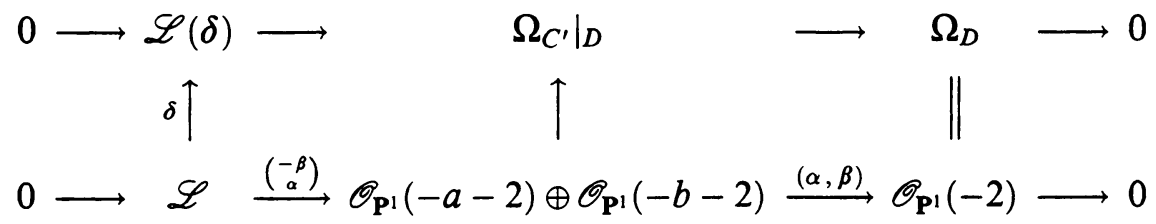

where $\mathscr{L}=\mathscr{O}_{\mathbf{P}^{1}}(-g-1)$, and it is obvious that the upper sequence splits iff $\delta \in I_{C}=(\alpha, \beta)$.

Corollary 2.4 provides another significant justification for using the name "Clifford index" for the invariant of a ribbon that we have defined:

We define a GENERALIZED LINEAR SERIES OF DEGREE $n$ AND DIMENSION $r$ on a ribbon $C$ to be an ordinary linear series (line bundle and space of sections) of degree $n-d$ on the blow-up of $C$ at some divisor $\delta$ of length $d$ in $D$. Note that blowing up $C$ corresponds to removing base points; if $C$ were smooth, we could remove base points without changing $C$, so that the given definition is a natural extension of the smooth case.

From Corollary 2.4 we can say that a ribbon of Clifford index $a$ has a generalized linear series of degree $a+2$ and dimension 1, corresponding to a linear series of Clifford index $a$ (in the usual sense!). For this we need only take 
the degree 2 map to $\mathbf{P}^{1}$ of the hyperelliptic $a$-fold blow-up guaranteed by the Corollary.

We shall see that there is a good notion of torsion-free sheaf on a ribbon corresponding to the notion of generalized linear series, and also that $C$ does not have any generalized linear series of dimension 1 and degree $n$ for $n<a+2$ - that is, none of Clifford index less than the Clifford index of the ribbon. The same is true of generalized linear series of higher dimension, as is proved in Eisenbud-Green [1995].

The same technique proves:

Corollary 2.5. The ideal $I_{C^{\prime}}$ corresponding to the blow-up $C^{\prime}$ of $C$ along $\delta$ is the "quotient"

$$
\left(I_{C^{\prime}}\right)=\left(I_{C}: I_{\delta}\right) \stackrel{\text { def }}{=}\left\{\gamma \in S \mid \gamma I_{\delta} \subseteq I_{C}\right\},
$$

where $I_{\delta}$ is the ideal in $S$ of the subscheme $\delta$.

In particular, when $\delta$ is a single point, we have

$$
\text { Cliff } C^{\prime}<\operatorname{Cliff} C
$$

iff either Cliff $=(g-1) / 2$ or $\delta$ is a zero of the (unique) lowest degree form in $I_{C}$.

Using the dual socle, we can make explicit the stratification of the set of ribbons $\mathbf{P}\left(\mathbf{H}^{0}\left(\mathscr{O}_{\mathbf{P}}(g-3)\right)\right)$ by Clifford index. The following necessary results are well-known in the theory of vector bundles. Let

$$
X \subset \mathbf{P}\left(\mathrm{H}^{0}\left(\mathscr{O}_{\mathbf{P}^{1}}(g-3)\right)\right)
$$

be the rational normal curve of one-quotients $\mathrm{H}^{0}\left(\mathscr{O}_{\mathbf{P}^{1}}(g-3)\right) \rightarrow k$ corresponding to evaluations at points of $\mathbf{P}^{1}$.

Proposition 2.6. Let $f$ be an element of $\mathbf{H}^{0}\left(\mathscr{O}_{\mathbf{P}^{1}}(g-3)\right)^{*}$, regarded as a point in $\mathbf{P}\left(\mathrm{H}^{0}\left(\mathscr{O}_{\mathbf{P}^{1}}(g-3)\right)\right)$. If $I$ is the ideal whose dual socle is $f$, then I contains a form of degree $\leq a$ iff $f$ lies in an a-secant $(a-1)$-plane to $X$.

Thus the set of ribbons of Clifford index $\leq a$ corresponds to the union of the $(a-1)$-planes $a$-secant to $X$. (Here we count limits of such planes as also being $a$-secant - for example tangent lines are considered 2-secant lines.)

Writing $f$ in terms of a basis $\left(s^{i} t^{g-3-i}\right)^{\star}$ of $\mathrm{H}^{0}\left(\mathscr{O}_{\mathbf{P}^{1}}(g-3)\right)^{*}$ dual to the monomial basis of $\mathrm{H}^{0}\left(\mathscr{O}_{\mathbf{P}}(g-3)\right)$ determined by the choice of coordinates $s, t$, say as

$$
f=\sum_{i=0}^{g-3} f_{i}\left(s^{i} t^{g-3-i}\right)^{\star}
$$

we can express the secant loci, and thus the sets of ribbons of Clifford index $\leq$ a given number, as special determinantal varieties:

Proposition 2.7. With notation as above, $f$ lies in an a-secant $(a-1)$-plane to $X$ iff the rank of the "catalecticant" matrix satisfies

$$
a \geq \operatorname{rank}\left(\begin{array}{cccc}
f_{0} & f_{1} & \ldots & f_{a-1} \\
f_{1} & f_{2} & \ldots & f_{a} \\
\ldots & \ldots & \ddots & \ldots \\
f_{b-1} & f_{b} & \ldots & f_{a+b-2}
\end{array}\right) .
$$


In fact the ideal of $a \times a$ minors of the catalecticant matrix is the whole homogeneous ideal of the corresponding secant locus, and this remains true for the $a \times a$ minors of any $a^{\prime} \times b^{\prime}$ catalecticant matrix

$$
\left(\begin{array}{cccc}
f_{0} & f_{1} & \ldots & f_{a^{\prime}-1} \\
f_{1} & f_{2} & \ldots & f_{a^{\prime}} \\
\ldots & \ldots & \ddots & \ldots \\
f_{b^{\prime}-1} & f_{b^{\prime}} & \ldots & f_{a^{\prime}+b^{\prime}-2}
\end{array}\right),
$$

as long as $a \leq a^{\prime}, b^{\prime}$; see Gruson-Peskine [1982] for a proof.

\section{RATIONAL RIBBONS 2: GluiNG}

Again in this section we shall consider ribbons $C$ on $D=\mathbf{P}^{1}$. We shall write $g$ for the genus of $C$.

Since ribbons on the affine line are all split, it is useful to regard ribbons on $D$ as being obtained by gluing together ribbons on the affine line. In this section we use this gluing to give another view of the classification of ribbons.

In the next section we shall exploit gluing to analyze line bundles and their sections, to locate the canonical bundle, and to form the canonical map of a ribbon to projective space.

We begin by fixing notation: we shall regard $D$ as glued together out of two open sets

$$
u_{1}=\operatorname{Spec} k[s], \quad u_{2}=\operatorname{Spec} k[t]
$$

via the identification $s^{-1}=t$ on $u_{1} \cap u_{2}$.

If $C$ is a ribbon on $D$, then by Corollary 1.3 , we may write

$$
\begin{aligned}
& \left.U_{1} \stackrel{\text { def }}{=} C\right|_{u_{1}} \cong \operatorname{Spec} k[s, \epsilon] / \epsilon^{2}, \\
& \left.U_{2} \stackrel{\text { def }}{=} C\right|_{u_{2}} \cong \operatorname{Spec} k[t, \eta] / \eta^{2},
\end{aligned}
$$

and $C$ may be specified by giving an appropriate gluing isomorphism between these two schemes over the set $u_{1} \cap u_{2}$.

Since the ideal sheaf $\mathscr{L} \cong \mathscr{O}_{P^{1}}(-g-1)$ of $D$ in $C$ is generated on $u_{1}$ by $\epsilon$ and on $u_{2}$ by $\eta$, and since the gluing isomorphism must restrict to the one already specified on $D$, we see at once that it can be written in the form:

$$
\begin{gathered}
\epsilon=t^{-g-1} \eta \\
s^{-1}=t+F(t) \eta
\end{gathered}
$$

on $u_{1} \cap u_{2}$, with some

$$
F(t) \in k\left[t, t^{-1}\right]=\mathscr{O}_{\mathbf{P}^{1}}\left(u_{1} \cap u_{2}\right) .
$$

Conversely, any such gluing data defines a ribbon of genus $\mathbf{g}$ on $\mathbf{P}^{1}$.

If we change the coordinates on $U_{1}$ and $U_{2}$ to $s^{\prime}$ and $t^{\prime}$ with

$$
s^{\prime}=s+p(s) \epsilon, \quad t=t^{\prime}+q(t) \eta,
$$


where $p(s)$ and $q\left(t^{\prime}\right)$ are polynomials, then we have

$$
\begin{aligned}
s^{\prime-1} & =s^{-1}-s^{-2} p(s) \epsilon \\
& =t+F(t) \eta+(t+F(t) \eta)^{2} p(s) t^{-g-1} \eta \\
& =t+\left(F(t)+t^{-g+1} p\left(t^{-1}\right)\right) \eta \\
& =t^{\prime}+q(t) \eta+\left(F\left(t^{\prime}+q(t) \eta\right)+\left(t^{\prime}+q(t) \eta\right)^{-g+1} p\left(\left(t^{\prime}+q(t) \eta\right)^{-1}\right)\right) \eta \\
& =t^{\prime}+\left(F(t)+t^{-g+1} p\left(t^{-1}\right)+q(t)\right) \eta,
\end{aligned}
$$

where we have repeatedly used the facts that

$$
s \eta=t^{-1} \eta, \quad t \eta=t^{\prime} \eta,
$$

etc. Also, if we multiply $s$ or $t$ by a scalar then $F$ will be multiplied by the same scalar. From this we see that $F$ is determined (at best) as an element of the projective space of lines in the quotient

$$
k\left[t, t^{-1}\right] /\left(k[t]+t^{-g+1} k\left[t^{-1}\right]\right) .
$$

On the other hand, using the covering of $\mathbf{P}^{1}$ by $u_{1}$ and $u_{2}$, this quotient may be identified via Čech cohomology as $\mathrm{H}^{1}\left(\mathscr{O}_{\mathbf{P}^{1}}(-g-1)\right)$. We have seen in the last section that the lines in this vector space classify the ribbons on $\mathbf{P}^{1}$. The main result of this section is that these two classifications are the same:

Theorem 3.1. Let $F \in k\left[t, t^{-1}\right]$ be a Laurent polynomial. If $C$ is the ribbon defined by gluing $U_{1}$ and $U_{2}$ as above, then $F$ is proportional to the class $e_{C}$ of the restricted cotangent sequence of $C$ in

$$
\mathrm{H}^{1}\left(\mathscr{O}_{\mathbf{P}^{1}}(-g+1)\right)=k\left[t, t^{-1}\right] /\left(k[t]+t^{-g+1} k\left[t^{-1}\right]\right) .
$$

We must exhibit a construction of the restricted cotangent sequence by gluing. On $U_{1}$ we have

$$
\left.\Omega_{C}\right|_{U_{1}}=\left(\mathscr{O}_{C} d s \oplus \mathscr{O}_{C} d \epsilon\right) / 2 \epsilon d \epsilon
$$

so that

$$
\left.\left(\left.\Omega_{C}\right|_{D}\right)\right|_{u_{1}}=\mathscr{O}_{D} d s \oplus \mathscr{O}_{D} d \epsilon,
$$

and the restricted cotangent sequence, restricted further to $u_{1}$, takes the form

$$
0 \rightarrow\langle d \epsilon\rangle \rightarrow\langle d \epsilon\rangle \oplus\langle d s\rangle \rightarrow\langle d s\rangle \rightarrow 0,
$$

where we have written $\langle x\rangle$ for the free module with basis element $x$. Of course we have a similar sequence, with $t$ and $\eta$ on $u_{2}$. On the intersection $u_{1} \cap u_{2}$ we have $\eta d t=\eta d \eta=0$ so

$$
d \epsilon=t^{-g-1} d \eta, \quad d s=-t^{-2} d t-t^{-2} F d \eta .
$$

Thus the gluing takes the form

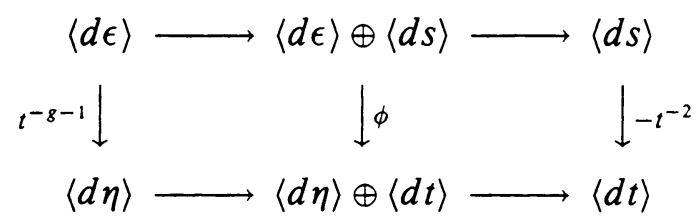

where we have written $\phi$ for the matrix

$$
\left(\begin{array}{cc}
-t^{-g-1} & -t^{-2} F \\
0 & -t^{-2}
\end{array}\right)
$$



in

To show that $F$ is proportional to the class of restricted cotangent sequence

$$
\mathbf{H}^{1}\left(\mathscr{O}_{\mathbf{P}^{1}}(-g+1)\right)=k\left[t, t^{-1}\right] /\left(k[t]+t^{-g+1} k\left[t^{-1}\right]\right),
$$

it suffices to show that $F$ is proportional to the image of $1 \in \mathrm{H}^{0} \mathscr{O}_{\mathbf{P}^{1}}$ under the connecting homomorphism

$$
\delta: \mathrm{H}^{0} \mathscr{O}_{\mathbf{P}^{1}} \rightarrow \mathrm{H}^{1} \mathscr{O}_{\mathbf{P}^{1}}(-g+1)
$$

induced by the restricted cotangent sequence twisted by $\mathscr{O}_{\mathbf{P}^{1}}(2)$.

The effect of the twist is to replace the gluing diagram $\left({ }^{*}\right)$ by the diagram

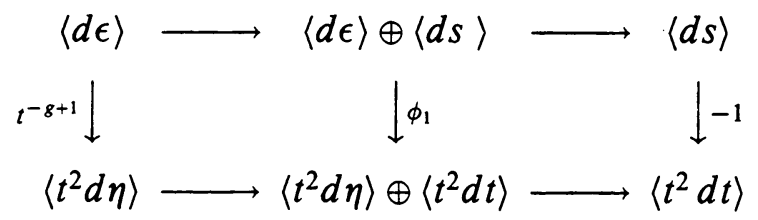

where now $\phi_{1}$ is the matrix

$$
\left(\begin{array}{cc}
-t^{-g+1} & -F \\
0 & -1
\end{array}\right)
$$

We may compute the connecting homomorphism $\delta$ from this gluing description: the Čech 1-cocyle representing $\delta(1)=\delta(d s) \in \mathrm{H}^{1}\left(\mathscr{O}_{\mathbf{P}^{1}}(-g+1)\right)$ is obtained by taking

$$
t^{2} d t-\phi_{1}(d s)
$$

as an element of

$$
\left.\mathscr{O}_{\mathbf{P}}(-g+1)\right|_{u_{1} \cap u_{2}} .
$$

Since this is $-F$, we are done.

Using the ideas developed in the last section, we may rephrase this result in terms of the ideal $I_{C}$ associated to the ribbon. The Laurent polynomial $F$ determines a linear form on the set of polynomials in $t$ by the rule

$$
h \mapsto \text { residue }_{t=0} F h \text {. }
$$

The usual duality between $\mathrm{H}^{1}\left(\mathscr{O}_{\mathbf{P}^{1}}(-g+1)\right)$ and $\mathrm{H}^{0}\left(\mathscr{O}_{\mathbf{P}^{1}}(g-3)\right)$ is given by restricting this functional to the polynomials of degree $\leq g-3$, and identifying these with the forms of degree $g-3$ in $s$ and $t$. In this way we may regard $F$ as a functional on $S_{d-3}$, and as such we have:

Corollary 3.2. $F$ is a dual socle generator for the ideal $I_{C}$ of the ribbon defined by $F$.

\section{LINE BUNDLES ON RIBBONS}

Again in this section we shall consider ribbons $C$ on $D=\mathbf{P}^{1}$, and we shall write $g$ for the genus of $C$.

In this section we shall explain how to classify the line bundles on a rational ribbon, and we shall compute the sections of a given line bundle. In the next section we shall apply these ideas to find and study the canonical bundle.

Unfortunately, we do not know a method of describing a line bundle on a ribbon $C$ in terms of some structure on the underlying $\mathbf{P}^{1}$ analogous to the 
method of the restricted cotangent sequence for describing $\mathscr{O}_{C}$ itself. Thus we shall use a gluing description analogous to the ideas developed in the last section. In particular, we shall make use of the notation developed in the last section for the gluing of $C$ by means of the Laurent polynomial $F$. However, we can take a few steps before using coordinates:

First we compute the Picard group of $C$ :

\section{Proposition 4.1.}

$$
\operatorname{Pic} C=\mathrm{H}^{1}\left(\mathscr{O}_{\mathbf{P}^{1}}(-g-1)\right) \times \mathbf{Z} \cong k^{g} \times \mathbf{Z},
$$

where the projection to the $\mathbf{Z}$ factor is given by associating to a line bundle $L$ the degree of the restriction $\left.L\right|_{D}$.

Proof. From the exact sequence

$$
0 \rightarrow \mathscr{L} \rightarrow \mathscr{O}_{C} \rightarrow \mathscr{O}_{D} \rightarrow 0
$$

we derive an "exponential sequence"

$$
0 \rightarrow \mathscr{L} \rightarrow \mathscr{O}_{C}^{*} \rightarrow \mathscr{O}_{D}^{*} \rightarrow 1
$$

by sending a local section $\sigma \in \mathscr{L}$ to $1+\sigma \in \mathscr{O}_{C}^{*}$. Taking cohomology, we get

$$
0 \rightarrow \mathrm{H}^{1}(\mathscr{L}) \rightarrow \operatorname{Pic} C \operatorname{Pic} D \rightarrow 1,
$$

where the last map represents restriction to $D$. Since $\operatorname{Pic} D \cong \mathbf{Z}$ via the degree and

$$
\mathrm{H}^{1}(\mathscr{L})=\mathrm{H}^{1}\left(\mathscr{O}_{\mathbf{P}}(-g-1)\right) \cong k^{g},
$$

this gives the desired conclusion.

As in the smooth case, the fundamental invariant of a line bundle $L$ is its degree, here defined as

From the RESTRICTION SEQUENCE

$$
\operatorname{deg} L \stackrel{\text { def }}{=} \chi(L)-\chi\left(\mathscr{O}_{C}\right) .
$$

$$
\left.0 \rightarrow \mathscr{L} L \rightarrow L \rightarrow L\right|_{D} \rightarrow 0
$$

for $L$ we see that the degree of $L$ may be computed from the knowledge of $\mathscr{L} L$ provided by the following result:

Proposition 4.2. If $L$ is a line bundle on $C$ and $\left.L\right|_{D} \cong \mathscr{O}_{\mathbf{P}^{1}}(n)$ then $\mathscr{L} L \cong$ $\mathcal{O}_{\mathrm{P}^{1}}(n-g-1)$, and $\operatorname{deg} L=2 n$.

Proof. Since $\mathscr{L}^{2}=0$ we have

$$
\mathscr{L} \otimes L=\left.\mathscr{L} \otimes L\right|_{D}=\mathscr{O}_{\mathbf{P}^{1}}(n-g-1) .
$$

On the other hand, we see by restriction to an affine open set that $\mathscr{L} L$ is a line bundle, so the epimorphism $\mathscr{L} \otimes L \rightarrow \mathscr{L} L$ must be an isomorphism. The degree computation now follows trivially.

To replace line bundles of odd degree, it seems that one must turn to torsionfree sheaves, which are line bundles on blowups of $C$, as we shall see later.

Next we turn to the question of sections of a line bundle. What we need is a formula for the connecting homomorphism

$$
\delta_{L}: \mathrm{H}^{0}\left(\left.L\right|_{D}\right) \rightarrow \mathrm{H}^{1}(\mathscr{L} L)
$$


associated to the restriction sequence. We shall get it in terms of gluing data for the line bundle.

To construct line bundles by gluing, note first that all line bundles on one of the sets $U_{i}$ are trivial (one can see this, for example, from the analogue of the exact sequence for the Picard group above, or by standard commutative algebra) so that to give a line bundle $L$ on $C$ whose restriction to $D$ is $\mathscr{O}_{\mathbf{P}^{1}}(n)$ it is enough to specify that

$$
\begin{aligned}
L & =k[s, \epsilon] e_{1} & & \text { on } U_{1}, \\
L & =k[t, \eta] e_{2} & & \text { on } U_{2}, \\
e_{1} & =(t+F \eta)^{n}(1+G \eta) e_{2} & & \text { on } U_{1} \cap U_{2}
\end{aligned}
$$

for some $G \in k\left[t, t^{-1}\right]$. Conversely, any $G \in k\left[t, t^{-1}\right]$ may be used in this way to construct a line bundle $L$ on $C$.

If we change coordinates on $U_{1}$ and $U_{2}$, say by

$$
e_{1}=(1+m(s) \epsilon)^{-1} e_{1}^{\prime}, \quad e_{2}=(1+n(t) \eta) e_{2}^{\prime},
$$

then we get

$$
\begin{aligned}
e_{1}^{\prime} & =(t+F \eta)^{n}(1+G \eta)\left(1+m(s) t^{-G-1} \eta\right)(1+n(t) \eta) e_{2}^{\prime} \\
& =(t+F \eta)^{n}\left(1+\left(G+m\left(t^{-1}\right) t^{-g-1}+n(t)\right) \eta\right) e_{2}^{\prime}
\end{aligned}
$$

so that to specify $L$ it is enough to give $G$ as an element of

$$
k\left[t, t^{-1}\right] /\left(k[t]+t^{-g-1} k\left[t^{-1}\right]\right)=\mathrm{H}^{1}\left(\mathscr{O}_{\mathbf{P}^{1}}(-g-1)\right) .
$$

We can now state the main result of this section:

Theorem 4.3. Let $L$ is a line bundle on $C$ of degree $2 n$ given by gluing data as above. If $p=p(t)$ is a polynomial of degree $\leq n$, so that $\left.p e_{2}\right|_{D}$ defines an element

$$
\sigma \in \mathrm{H}^{0}\left(\left.L\right|_{D}\right)=\mathrm{H}^{0}\left(\mathscr{O}_{\mathbf{P}^{1}}(n)\right),
$$

then

$$
\begin{aligned}
\delta_{L}(\sigma) & =-\left(p^{\prime} F+p G\right) \\
& \in k\left[t, t^{-1}\right] /\left(k[t]+t^{n-g-1} k\left[t^{-1}\right]\right) \\
& =\mathrm{H}^{1}\left(\mathscr{O}_{\mathbf{P}^{1}}(n-g-1)\right)
\end{aligned}
$$

where $p^{\prime}=\partial p(t) / \partial t$.

Further, the space of sections of $L$ restricted to $U_{2}=\operatorname{Spec} k\left[t, t^{-1}\right]$ is the direct sum of the space of elements $q(t) \eta$, for $q$ a polynomial of degree $\leq$ $n-g-1$, and the space of expressions of the form $p(t)+p_{1}(t) \eta$ where $p(t)$ is a polynomial of degree $\leq n$ satisfying $\delta_{L}(\sigma)=0$ and $p_{1}(t) \in k[t]$ is the "polynomial part" of $p^{\prime} F+p G$, that is,

$$
p_{1}(t) \equiv p^{\prime}(t) F(t)+p(t) G(t) \quad \bmod t^{-1} k\left[t^{-1}\right] .
$$

Proof. The connecting homomorphism $\left.H^{0} L\right|_{p} \rightarrow H^{1}(\mathscr{L} L)$ is obtained by comparing liftings of $\sigma$ on $U_{1}$ and on $U_{2}$. We have

$$
\left.p(t) e_{2}\right|_{u_{1} \cap u_{2}}=\left.p\left(s^{-1}\right) s^{n} e_{1}\right|_{u_{1} \cap u_{2}}
$$

so that $\delta_{L}(\sigma)$ is given as a Čech cocycle by the element

$$
p(t) e_{2}-p\left(s^{-1}\right) s^{n} e_{1} \in \mathscr{L} L\left(U_{1} \cap U_{2}\right)=\left.\mathscr{O}_{\mathbf{P}^{1}}(n-g-1)\right|_{u_{1} \cap u_{2}} .
$$


Using the gluing formulas we get

$$
\begin{aligned}
p(t) e_{2}-p\left(s^{-1}\right) s^{n} e_{1} & =p(t) e_{2}-p(t+F \eta)(t+F \eta)^{-n}(t+F \eta)^{n}(1+G \eta) e_{2} \\
& =p(t) e_{2}-p(t+F \eta)(1+G \eta) e_{2} \\
& =p(t) e_{2}-\left(p(t)+p^{\prime}(t) F \eta\right)(1+G \eta) e_{2} \\
& =-\left[p(t) G+p^{\prime}(t) F\right] \eta e_{2},
\end{aligned}
$$

which gives the desired formula for $\delta_{L}$.

The elements $q(t) \eta e_{2}$, with $q$ a polynomial of degree $\leq n-g-1$, clearly represent the sections of $\mathscr{L} L$. The rest of the sections of $L$ are obtained by lifting sections $\sigma$ of $\left.L\right|_{D}$ that go to 0 under $\delta_{L}$. Given such a section, represented say by $p(t) e_{2}$ on $u_{2}$ as above, we must find an expression

$$
p(t)+p_{1}(t) \eta
$$

that is equal in $k\left[t, t^{-1}, \eta\right]=k\left[s, s^{-1}, \epsilon\right]$ to some element coming from $k[s, \epsilon] e_{1}$. But we have just shown that

$$
p(t) e_{2}-p\left(s^{-1}\right) s^{n} e_{1}=-\left[p(t) G+p^{\prime}(t) F\right] \eta e_{2} .
$$

Take $p_{1}$ as in the theorem. Setting

$$
r(t) \eta e_{2}=\left(p(t)+p_{1}(t) \eta\right) e_{2}-p\left(s^{-1}\right) s^{n} e_{1},
$$

we see that $r(t)$ will have no polynomial part. That is, $r(t) \in k\left[t^{-1}\right]$. In addition, if $\delta_{L} \sigma=0$ then $r(t) \in t^{n-g-1} k\left[t^{-1}\right]$. Thus

$$
\begin{aligned}
r(t) \eta e_{2} & =r\left(s^{-1}\right)\left(s^{-g-1} \epsilon\right) s^{n} e_{1} \\
& =s^{n-g-1} r\left(s^{-1}\right) \in k[s] \epsilon e_{1},
\end{aligned}
$$

so that $\left(p(t)+p_{1}(t) \eta\right) e_{2}$ represents a section as claimed.

\section{THE CANONICAL EMBEDDING}

Again in this section we shall consider ribbons $C$ on $D=\mathbf{P}^{1}$, and we shall write $g$ for the genus of $C$. We shall continue to use the notation introduced in the previous section.

As an application of the work done in the previous section, we can determine the canonical line bundle and its sections. Note that a ribbon is locally Gorenstein, so that the canonical sheaf really is a line bundle, and has degree $2 g-2$ by the Riemann-Roch formula.

If $G(t)=\sum_{-n}^{n} a_{i} t^{i} \in k(t)$ is a rational function, then we write

$$
G_{\text {polynomial }} \stackrel{\text { def }}{=} \sum_{0}^{n} a_{i} t^{i}
$$

for the "polynomial part" of $\mathrm{G}$.

Theorem 5.1. The restriction sequence of the canonical line bundle $K_{C}$ has the form

$$
0 \rightarrow \mathscr{O}_{\mathbf{P}}(-2) \rightarrow K_{C} \rightarrow \mathscr{O}_{\mathbf{P}^{1}}(g-1) \rightarrow 0 .
$$

The associated connecting homomorphism $\delta_{C}$ is 0 , so that the induced linear series on $D$ is the complete series of degree $g-1$. The gluing data of the canonical bundle of $C$ is given by

$$
G=\partial F / \partial t,
$$


so that the sections of $K_{C}$ are represented by the expressions

$$
p+(\partial(p F) / \partial t)_{\text {polynomial }} \eta
$$

where $p$ ranges over the polynomials of degree $\leq g-1$ in $t$. Thus if we take $F$ of the form

$$
F(t)=\sum_{i=1}^{g-2} F_{i} t^{-i}
$$

then the elements of a basis of global sections of $K_{C}$ restrict on $U_{2}$ to the elements

$$
t^{i}+\sum_{j=0}^{i-2}(j+1) F_{i-j-2} t^{j} \eta \quad(i=0, \ldots, g-1) .
$$

Proof. The form of the restriction sequence comes simply from the degree of $K_{C}$, and thus from Riemann-Roch. From Riemann-Roch we also know that $\mathrm{h}^{0}\left(K_{C}\right)=g$. Since $\mathrm{h}^{0}\left(\mathscr{O}_{\mathbf{P}^{1}}(g-1)\right)=g$ while $\mathrm{h}^{0}\left(\mathscr{O}_{\mathbf{P}^{1}}(-2)\right)=0$, this implies that

$$
\delta_{K_{C}}: \mathrm{H}^{0}\left(\mathscr{O}_{\mathbf{P}^{1}}(g-1)\right) \rightarrow \mathrm{H}^{1}\left(\mathscr{O}_{\mathbf{P}^{1}}(-2)\right)
$$

is 0 . Further, since no other line bundle of degree $2 g-2$ has as many sections as the canonical bundle, this vanishing actually characterizes $K_{C}$.

By the formula for $\delta_{K_{C}}$ given in Theorem 4.3, $\delta_{K_{C}}=0$ means that if $G=$ $G(t)$ is the gluing data for $K_{C}$, then

$$
\text { residue }_{t=0} p^{\prime} F+p G=0
$$

for all polynomials $p=p(t)$ of degree $\leq g-1$. Since the residue of the derivative of a rational function is automatically 0 , this can be achieved by taking

$$
G=\partial F / \partial t
$$

so that

$$
p^{\prime} F+p G=\partial(p F) / \partial t .
$$

By the remark above characterizing $K_{C}$, this establishes the formula for $G$ given in the Theorem. The rest of the Theorem follows by direct computation from Theorem 4.3.

We next wish to show that the canonical linear series defines an embedding of $C$ as an arithmetically Cohen-Macaulay (even Gorenstein) subscheme of $\mathbf{P}^{\boldsymbol{g}-1}$. As a first step we have:

Corollary 5.2. The canonical series on $C$ is base point free.

Proof. It induces the complete series on $D$.

The following is the main result of this section. It continues the strict analogy with the theory of smooth curves, and is the first stage of what might be called "Green's conjecture for ribbons", which will be discussed below.

Theorem 5.3 (Noether's Theorem for Ribbons). Let the genus of $C$ be $g \geq 2$. If $C$ is hyperelliptic, then the canonical map is the degree 2 projection onto $\mathbf{P}^{1}$ composed with the embedding of $\mathbf{P}^{1}$ into $\mathbf{P}^{g-1}$ as the rational normal curve. 
If $C$ is not hyperelliptic, then the canonical series embeds $C$ as an arithmetically Gorenstein subscheme of $\mathbf{P}^{g-1}$.

Proof. If $C$ is hyperelliptic, then the form of the canonical sections given in Theorem 5.1 makes it clear that the canonical image of $C$ is the rational normal curve of degree $g-1$. In particular, the map of $C$ onto this image is 2 to 1 , and since $g \geq 2$ there is only one such map (see the remark at the beginning of section 2). Conversely, we see that if the canonical image of $C$ is the rational normal curve of degree $d-1$, then $C$ is hyperelliptic.

Now suppose that $C$ is not hyperelliptic. It is enough to show that the canonical series defines an embedding of $C$ as an arithmetically Cohen-Macaulay curve; the fact that the embedding line bundle is the canonical bundle then implies that the image is arithmetically Gorenstein.

Since the restriction of the canonical series to $D \subset C$ is the complete series, it defines an embedding of $D$ as an arithmetically Cohen-Macaulay curve. Writing $\mathscr{J}_{D}$ for the ideal sheaf of $D \subset \mathbf{P}^{g-1}$, it is enough to show that the induced maps

$$
\mathrm{H}^{0}\left(\mathscr{I}_{D} / \mathscr{I}_{D}^{2}(n)\right) \rightarrow \mathrm{H}^{0}(\mathscr{L}(n))
$$

are onto for all $n$.

The following well-known lemma identifies the sheaf involved:

Lemma 5.4. The conormal bundle to the rational normal curve $D \subset \mathbf{P}^{r}$ of degree $g-1$ is

$$
\mathscr{I}_{D} / \mathscr{I}_{D}^{2} \cong \mathrm{H}^{0}\left(\mathscr{O}_{\mathbf{P}^{1}}(g-3)\right) \otimes \mathscr{O}_{\mathbf{P}^{1}}(-g-1)
$$

equivariantly for the action of $\mathrm{SL}(2)$.

Proof of Lemma 5.4. This is just the dual of a special case of Proposition 5A.2.

Returning to the proof of Theorem 5.3, we see that the natural map $\mathscr{I}_{D} / \mathscr{I}_{D}^{2}$ $\rightarrow \mathscr{L}$ has the form

$$
\mathscr{I}_{D} / \mathscr{I}_{D}^{2} \cong \mathscr{O}_{\mathbf{P}^{1}}(-(g-1))^{g-2} \stackrel{\phi}{\longrightarrow} \mathscr{O}_{\mathbf{P}^{1}}(-(g-1)) \cong \mathscr{L}
$$

and is thus either identically 0 or a split epimorphism. The image of this map is in either case $\mathscr{I}_{D} / \mathscr{I}_{C}$, where $\mathscr{I}_{C}$ is the ideal sheaf of $C$. In the latter case we are done by Theorem 1.8. In the former case we see that $\mathscr{I}_{D}=\mathscr{I}_{C}$, so that the canonical image of $C$ is the rational normal curve, and $C$ is hyperelliptic by the remarks above, contradicting our assumption.

As was pointed out to us by Joe Harris, one can also give a proof of "Noether's theorem for ribbons" along the lines of Noether's original proof: First one may use Riemann-Roch to estimate the number of conditions imposed by a subscheme of length 2 and thus show that the canonical map is a closed immersion.

Next one checks the number of quadratic (and then cubic ...) equations satisfied by the curve by checking the number of conditions imposed on quadrics by the general hyperplane section. For a reduced nonhyperelliptic curve of genus $g$ the general hyperplane section of the canonical embedding consists of $2 g+2$ points in linearly general position in $\mathbf{P}^{g-2}$, and it is easy to see that such a set of points imposes at least $2 g+1$ conditions on quadrics. In the case of a ribbon, the hyperplane section consists of $g+1$ double points. But these are again in general position in a suitable sense, as one may show by a monodromy argument; see for example Eisenbud-Harris [1992] and, for a more general study, Chandler [1995]. 
We isolate for future use a piece of information from the proof of Theorem 5.3:

Corollary 5.5. If $C \subset \mathbf{P}^{g-1}$ is a nonhyperelliptic ribbon in its canonical embedding, and $\mathscr{I}_{C} \subset \mathscr{I}_{D}$ are its ideal sheaf and that of the underlying rational normal curve $D=\mathbf{P}^{1}$, then

$$
\mathscr{I}_{D} / \mathscr{I}_{C} \cong \mathscr{O}_{\mathbf{P}^{1}}(-g-1)
$$

is a direct summand of the conormal bundle

$$
\mathscr{I}_{D} / \mathscr{I}_{D}^{2} \cong \mathscr{O}_{\mathbf{P}^{1}}(-g-1)^{g-2} \text {. }
$$

We remark that the proof of the Theorem indicates a third view of the classification of ribbons: To specify a nonhyperelliptic ribbon of genus $\mathrm{g}$ in its canonical embedding one must simply give a corank 1 direct summand of the conormal bundle of the rational normal curve of degree $g-1-$ this subbundle, together with the square of the ideal of the rational normal curve, generates the ideal of the ribbon. By Lemma 5.4, such a summand is specified by an element of the dual of $\mathrm{H}^{0}\left(\mathscr{O}_{\mathbf{P}^{1}}(g-3)\right)$, up to scalars. This is the same element that specifies the gluing data or the dual socle generator, as the reader may check.

\section{CANONICAL RIBbons ARE SMOOTh POINTS OF Hilb}

Again in this section we shall consider ribbons $C$ on $D=\mathbf{P}^{1}$, and we shall write $g$ for the genus of $C$.

In this section we shall show that ribbons all lie in the smooth locus of the Hilbert scheme of canonical curves. The result is from joint work with Joe Harris.

Theorem 6.1. If $C \subset \mathbf{P}^{g-1}$ is a nonhyperelliptic ribbon in its canonical embedding, then $C$ represents a smooth point of the Hilbert scheme of curves of genus $g$ and degree $2 g-2$, lying on a component of dimension $(3 g-3)+\left(g^{2}-1\right)$.

The given dimension is of course also the dimension of the component containing the smooth curves, and in fact Fong [1993] shows that they are the same.

Proof. Let $D=C_{\text {red }} \cong \mathrm{P}^{1}$ as usual, and write $I_{C}$ and $\mathscr{I}_{D}$ for the ideal sheaves of $C$ and $D$ in the canonical embedding of $C$ in $\mathbf{P}^{g-1}$. Let

$$
N_{C}=\operatorname{Hom}_{C}\left(\mathscr{I}_{C} / \mathscr{I}_{C}^{2}, \mathscr{O}_{C}\right)
$$

be the normal sheaf.

Because $C$ is locally a complete intersection, $N_{C}$ is a vector bundle of rank $g-2$ on $C$ and it suffices to show that $\mathrm{h}^{0}\left(N_{C}\right)=g^{2}+3 g-4$ and $\mathrm{h}^{1}\left(N_{C}\right)=0$ (see for example Sernesi [1986, Corollaries 8.5 and 8.6].) To do this, we shall use the restriction sequence

$$
\left.\left.0 \rightarrow N_{C}\right|_{D} \otimes \mathscr{O}_{\mathbf{P}^{1}}(-g-1) \rightarrow N_{C} \rightarrow N_{C}\right|_{D} \rightarrow 0,
$$

and we must determine the bundle $\left.N_{C}\right|_{D}$. Since $\mathscr{I}_{C} / \mathscr{I}_{C}^{2}$ is a vector bundle, the operations of taking its dual and restricting it to $D$ commute, and we get

$$
\begin{aligned}
\left.N_{C}\right|_{D} & =\left.\operatorname{Hom}_{C}\left(\mathscr{I}_{C} / \mathscr{I}_{C}^{2}, \mathscr{O}_{C}\right)\right|_{D} \\
& =\operatorname{Hom}_{D}\left(\left.\mathscr{I}_{C}\right|_{D} /\left.\mathscr{I}_{C}\right|_{D} ^{2}, \mathscr{O}_{D}\right) \\
& =\operatorname{Hom}_{D}\left(\mathscr{I}_{C} / \mathscr{I}_{C} \mathscr{I}_{D}, \mathscr{O}_{D}\right) .
\end{aligned}
$$


By Corollary 5.6, $\mathscr{I}_{C} / \mathscr{I}_{D}^{2} \cong \mathscr{O}_{\mathbf{P} 1}(-g-1)^{g-3}$, so $\operatorname{Hom}_{D}\left(\mathscr{I}_{C} / \mathscr{I}_{C} \mathscr{I}_{D}, \mathscr{O}_{D}\right)$ has a subbundle isomorphic to $\mathscr{O}_{\mathbf{P}^{1}}(g+1)^{g-3}$. To compute the quotient, note that it is a line bundle; thus to make the computation, it suffices to know the first chern class of $\left.N_{C}\right|_{D}$.

Again because $C$ is locally a complete intersection, we may calculate the chern class of $N_{C}$ just as we would in the case where $C$ is smooth: that is, we have

$$
\begin{aligned}
\mathscr{O}_{C}(1) & =\omega_{C}=\wedge^{g-2} N_{C} \otimes \omega_{\mathbf{P}^{g-1}} \\
& =\wedge^{g-2} N_{C} \otimes \mathscr{O}_{\mathbf{P}^{g-1}}(-g)=\wedge^{g-2} N_{C} \otimes \mathscr{O}_{C}(-g),
\end{aligned}
$$

so

$$
c_{1}\left(N_{C}\right)=c_{1}\left(\wedge^{g-2} N_{C}\right)=\mathscr{O}_{C}(g+1) .
$$

Of course the restriction of the first chern class is the first chern class of the restriction, so

$$
c_{1}\left(\left.N_{C}\right|_{D}\right)=\mathscr{O}_{D}(g+1)=\mathscr{O}_{\mathbf{P}^{1}}((g+1)(g-1)) .
$$

Subtracting the first chern class of the subbundle we already know, we see that $N_{C} \mid D$ fits into an exact sequence

$$
\left.0 \rightarrow \mathscr{O}_{\mathbf{P}^{1}}(g+1)^{g-3} \rightarrow N_{C}\right|_{D} \rightarrow \mathscr{O}_{\mathbf{P}^{1}}(2 g+2) \longrightarrow 0 .
$$

Putting the exact sequences $(*)$ and $(* *)$ together, we see at once that

$$
\mathrm{h}^{0}\left(N_{C}\right)=g^{2}+3 g-4, \quad \mathrm{~h}^{1}\left(N_{C}\right)=0,
$$

as required.

\section{SURFACES CONTAINING RIBBONS}

Again in this section we shall consider ribbons $C$ on $D=\mathbf{P}^{1}=\mathbf{P}_{k}^{1}$, and we shall write $g$ for the genus of $C$. Moreover, we assume that $k$ is algebraically closed.

In a certain sense the singularities of a ribbon are quite mild: they are "locally planar", so that for example a ribbon is locally a complete interesection (in any embedding).

However, the fact that every point is singular leads to some significant differences from the theory of reduced curves with locally planar singularities. In this section we prove a theorem that highlights such a difference. While any reduced projective curve with locally planar singularities is contained in many smooth surfaces, this is not the case for ribbons:

Theorem 7.1. Up to isomorphism of affine neighborhoods, the only pair $C \subset S$ where $C$ is a nonhyperelliptic ribbon and $S$ is a surface that is smooth along $C$, is the double conic in the projective plane.

Proof. If $D=C_{\text {red }} \subset S$, with $S$ a smooth surface, then the ideal sheaf $\mathscr{L}$ of $D \subset C$ is the conormal bundle of $D$ in $S$. If the arithmetic genus of $C$ is $g$ then $\operatorname{deg} \mathscr{L}=g+1$, so $D^{2}=g+1$. If $g \leq 2$ then $C$ is automatically hyperelliptic, so it suffices to treat the case $g \geq 3$. Hartshorne's Theorem on curves of high self-intersection [1969, Theorem 4.1] says that if $D^{2} \geq 4$ genus $(D)+5=5$, then either $D \subset S$ is a nonsingular cubic in the projective plane, or else $S$ is 
ruled and $D$ is a section. In the former case, $D$ would not be rational. In the latter case there is a projection from $S$ back to $D$ that induces a morphism $C \rightarrow D$, showing that $C$ is hyperelliptic. This proves Theorem 7.1 in the case $D^{2}=g+1 \geq 5$.

It remains to treat the case $D^{2}=4, g=3$. We claim that a smooth rational curve $D$ of self-intersection 4 on a smooth surface $S$ is (up to equivalence of affine neighborhoods) either a smooth conic in the plane or a section on one of the rational ruled surfaces $F_{0}, F_{2}, F_{4}$. This suffices to prove Theorem 7.1, since in the latter case the projection from $S$ to $D$ defines a a two-to-one map from $C$ to $D$, so that $C$ is hyperelliptic.

The claimed result is certainly known to experts on surfaces, but for want of a reference we sketch a proof. Let $K_{S}$ be the canonical divisor class of $S$. Since $D$ is rational, we see from the adjunction formula that $K_{S} \cdot D=-6$. Thus no multiple of $K_{S}$ can be effective. By the Enriques classification, $S$ is rational or ruled. If $S$ is ruled with base $B$, then since $D$ has positive self-intersection it cannot be contained in a fiber; thus $B$ is rational, so $S$ is rational in any case. In particular, $\chi\left(\mathscr{O}_{S}\right)=1$.

Now $H^{0}\left(\mathscr{O}_{S}\left(K_{S}\right)\right)=0$, and thus

$$
H^{2}\left(\mathscr{O}_{S}(D)\right)=H^{0}\left(\mathscr{O}\left(K_{S}-D\right)\right)=0 .
$$

By the Riemann-Roch formula on $S$,

$$
H^{0}\left(\mathscr{O}_{S}(D)\right) \geq D \cdot(D-K) / 2+\chi\left(\mathscr{O}_{S}\right)=6 .
$$

The restriction of $\mathscr{O}_{S}(D)$ to $D$ has degree 4 , and thus the associated line bundle $\mathscr{O}_{D}(D)$ has 5 independent global sections. The exact sequence

$$
0 \rightarrow H_{S}^{0} \rightarrow H^{0}\left(\mathscr{O}_{S}(D)\right) \rightarrow H^{0}\left(\mathscr{O}_{D}(D)\right)
$$

shows that $h^{0}\left(\mathscr{O}_{S}(D)\right)=6$, and the restriction map $H^{0}\left(\mathscr{O}_{S}(D)\right) \rightarrow H^{0}\left(\mathscr{O}_{D}(D)\right)$ is surjective.

It follows that the complete linear series $|D|$ associated to $D$ on $S$ has no base points on $D$, and thus no base points anywhere; it defines a morphism $\phi$ from $S$ to $\mathbf{P}^{5}$ of degree 4 . Since $\phi(S)$ is nondegenerate it cannot have degree $<4$, so we see that $\phi$ is birational, that its image is a surface of degree 4 in $\mathbf{P}^{4}$. Since the self-intersection of $D$ is the same as the self-intersection of the the hyperplane section of $\phi(S)$ that is the image of $D$, we see that $\phi$ does not blow down any curves meeting $D$. That is, $\phi$ is biregular in a neighborhood of $D$, so we may assume that $S=\phi(S) \subset \mathbf{P}^{5}$ from the outset.

According to the Del Pezzo-Bertini classification of surfaces of minimal degree, $S$ is either a ruled surface $F_{0}$ or $F_{2}$, or a cone over the rational normal quartic, which away from the vertex is $F_{4}$ or $S$ is the Veronese embedding of $\mathbf{P}^{2}$ in $\mathbf{P}^{5}$; see for example Eisenbud-Harris [1987]. In the former cases $D$ is a section of $S$ (not containing the vertex, in the case where $S$ is a cone) so $C$ is hyperelliptic by the argument above. In the latter case $C \subset S$ is isomorphic to the conic in the plane, as required.

Of course any ribbon can, by Bertini's theorem, be embedded in a surface with only isolated singularities, and these can be kept away from any finite set of points on the ribbon. It would be interesting to know more about the number and type of singularities that a ribbon imposes on a surface containing it. (Our 
attention was drawn to this question by $M$. Boratyńsky.) The easiest way to produce a surface containing a given ribbon in projective space is to project the ribbon from a point, and take the cone over the image. This image is the canonical image of another ribbon:

Theorem 7.2. If $C \subset \mathbf{P}^{g-1}$ is a ribbon in its canonical embedding, and $p \in C$ is a reduced point on $C$, then the image of $C$ under projection from $p$ is the image of the canonical map from the ribbon $C^{\prime}$ obtained by blowing up $C$ at $p$.

Proof. Projection defines a morphism from the blow-up of $\mathbf{P}^{g-1}$, and thus also from the blow-up $C^{\prime}$ of $C$. The restriction of this morphism to $C_{\text {red }}=$ $C_{\text {red }}^{\prime}=\mathbf{P}^{1}$ is given by the complete series of degree $g-2$. It follows that the corresponding series on $C^{\prime}$ has degree $2(g-1)-2$ and (linear) dimension $g-1$; since the genus of $C^{\prime}$ is $g-1$, this must by Riemann-Roch be the canonical series.

As a consequence we see inductively that the Clifford index is related to the length of the 2-linear part of the free resolution of a canonical ribbon, at least by an inequality. The result is the "easy half of the canonical ribbon conjecture":

Corollary 7.3. The 2-linear part of the free resolution of a canonical ribbon of genus $g$ and Clifford index $c$ has length at least $g-2-c$.

Proof. The 2-linear part of the resolution of a subscheme of projective space is always at least as long as the 2-linear part of the resolution of any subscheme containing it. Since the resolution of the cone in $\mathbf{P}^{g-1}$ over a subscheme $C^{\prime}$ of $\mathbf{P}^{g-2}$ has the same graded Betti numbers as the resolution of $C^{\prime}$ in $\mathbf{P}^{g-2}$, and since the blow-up of a nonhyperelliptic ribbon $C$ at a suitable point will have Clifford index one less than that of $C$ by Corollary 2.5 , we are inductively reduced to the case of the resolution of the canonical image of a hyperelliptic ribbon, which is of course the rational normal curve. But in this case - the case $c=0$-the resolution is well known (it is given by the Eagon-Northcott complex) and the result is true.

More concretely, the method shows that a ribbon of genus $g$ and Clifford index $a$ is contained in the cone over a rational normal curve of degree $g-1-a$, and thus that the free resolution of the ribbon contains that of the rational normal curve.

A simple example will illustrate the results of this section:

Example. The canonical ribbon of genus 4. Let $C \subset \mathbf{P}^{3}$ be a canonically embedded ribbon of genus 4 . The Clifford index of $C$ is necessarily 1 , and $C$ is the complete intersection of a cubic and quadric. The ideal $I_{C}$ associated to $C$ as in section 2 is generated by a linear form and quadratic form on $\mathbf{P}^{1}$. By choosing coordinates $s, t$ appropriately we may assume that $I_{C}=\left(s, t^{2}\right)$. It follows that the blow-up of $C$ at the reduced point $p$ given by $s=0$ in $C_{\text {red }}$ is a hyperelliptic ribbon. By Theorem 7.2, the image of $C$ under projection from $p$ is the reduced conic in $\mathbf{P}^{2}$. The ribbon thus lies on the cone over this conic, and this is the unique quadric containing $C$.

Choosing coordinates on $\mathbf{P}^{3}$ appropriately we may assume that $C_{\text {red }}$ is the 
rational normal curve $D$ defined by the $2 \times 2$ minors of the matrix

$$
\left(\begin{array}{lll}
x_{0} & x_{1} & x_{2} \\
x_{1} & x_{2} & x_{3}
\end{array}\right)
$$

and the quadric cone containing $C$ is given by the equation $x_{0} x_{2}-x_{1}^{2}=0$, while $C$ itself is closure in the cone of the "double" of the divisor that is $2 D$ away from the vertex of the cone ( $D$ is not Cartier at the vertex). The cubic form necessary to generate the homogeneous ideal of $C$ (which is unique modulo the ideal of $D$ ) may be written as the determinant of the matrix

$$
\left(\begin{array}{ccc}
x_{0} & x_{1} & x_{2} \\
x_{1} & x_{2} & x_{3} \\
x_{2} & x_{3} & 0
\end{array}\right)
$$

The corresponding cubic surface has a double line, and a total of 4 singular points on the rational normal curve, none of them at the vertex of the quadric cone; the general cubic in the ideal of $C$ has 4 singular points, all on the rational normal curve, bearing out Theorem 7.1.

\section{Free Resolutions}

We assume for simplicity that the ground field $k$ has characteristic 0 .

The material in this section was partially developed in conversation with Frank Schreyer and Joe Harris.

It turns out that all canonically embedded rational ribbons of given genus and Clifford index are hyperplane sections of a single surface, which is itself a ribbon on a rational normal scroll. This surface has the same numerical invariants as a smooth K3 surface; Following terminology suggested by Frank Schreyer, we shall call it a K3 carpet. In this section we explain the construction of $\mathrm{K} 3$ carpets, and we prove that the natural embedding of a $\mathrm{K} 3$ carpet of sectional genus $g$ into $\mathbf{P}^{g}$ is arithmetically Cohen-Macaulay. It follows that all ribbons of given genus and Clifford index have minimal free resolutions with the same graded betti numbers; in particular, the canonical ribbon conjecture is true for all of them if it is true for one. To check the conjecture, it would suffice to compute a minimal free resolution of each $\mathrm{K} 3$ carpet.

In this secction we shall compute a nonminimal resolution of a $\mathrm{K} 3$ carpet, and we shall explain how to measure its nonminimality in terms of certain maps of vector spaces defined by elementary multilinear algebra. In particular, we explain some conjectures that would imply the canonical ribbon conjecture.

The description of the nonminimal resolution is facilitated by the observation that any $\mathrm{K} 3$ carpets is an anticanonical divisor on a certain (reduced) 3-fold (depending only on the genus and degree of the plane section of the $\mathrm{K} 3$ carpet) that thus appears as a degenerate Fano 3 -fold $J$ in $\mathbf{P}^{g+1}$. The Fano 3 -fold $J$ is extremely easy to describe: is simply the join variety of a pair of rational normal curves. It is equally easy to describe its minimal free resolutions $F$, which is the tensor product of two Eagon-Northcott complexes. The minimal free resolution of the canonical line bundle $\omega_{J}$ on this Fano 3-fold is $F^{*}$, the dual of $F$, up to a shift in degrees. It follows easily that a (nonminimal) free resolution of a K3 carpet may be constructed as a mapping cone of a map of complexes $F^{*} \rightarrow F$. This map is only unique up to homotopy, but it has a 
canonical representative that is equivariant with respect to the $S L(2)$ actions on the two rational normal curves. Most of the work in this section is devoted to an explicit construction of this canonical representative.

Let $S(a, g-1-a)$ be a rational normal scroll, the union of lines joining corresponding points on a rational normal curve of degree $a$ and a rational normal curve of degree $g-1-a$ in $\mathbf{P}^{g}$ According to Hulek-Van de Ven [1985] there is a unique double structure $X$ on $S(a, g-1-a)$, a ribbon on $S(a, g-1-a)$ in the sense of section 1 of this paper, with trivial canonical bundle. We shall call this double structure a $\mathrm{K} 3$ carpet. We give a construction of this carpet below.

Fix integers $1 \leq a \leq b$ and let $g=a+b+1$. In $\mathbf{P}^{g}$ we consider a pair of disjoint linear spaces $\mathbf{P}^{a}$ and $\mathbf{P}^{b}$, and the rational normal curves $D_{a} \subset \mathbf{P}^{a}$ and $D_{b} \subset \mathbf{P}^{b}$. Let $J$ be the join of these two rational normal curves; that is, $J$ is the union of the lines joining points of $D_{a}$ to points of $D_{b}$. The K3 carpet $X$ in which we are interested lies as an anticanonical divisor on $J$.

If we write $R_{a}$ and $R_{b}$ for the homogeneous coordinate rings of $D_{a}$ and $D_{b}$ in $\mathbf{P}^{a}$ and $\mathbf{P}^{b}$ respectively, then the homogeneous coordinate ring of $J$ in $\mathbf{P}^{g}$ is $R_{J}=R_{a} \otimes_{k} R_{b}$, graded by total degree. We regard $R_{a}$ and $R_{b}$ as homomorphic images of polynomial rings $T_{a}$ and $T_{b}$ in $a+1$ and $b+1$ variables, respectively.

Writing $\omega_{a}$ and $\omega_{b}$ for the canonical modules of $R_{a}$ and $R_{b}$, the canonical module of $R_{J}$ is given by $\omega_{J}=\omega_{a} \otimes_{k} \omega_{b}$. Further, we have

$$
\operatorname{Hom}_{R_{J}}\left(\omega_{J}, R_{J}\right)=\operatorname{Hom}_{R_{a}}\left(\omega_{a}, R_{a}\right) \otimes_{k} \operatorname{Hom}_{R_{b}}\left(\omega_{b}, R_{b}\right) \text {. }
$$

Identifying $D_{a}$ with $\mathbf{P}^{1}=\mathbf{P}(V)$, where $V$ is a 2-dimensional vector space, we have $\omega_{a}=\mathscr{O}_{\mathbf{P} 1}(-2)$, and a moment's argument gives

$$
\operatorname{Hom}_{R_{a}}\left(\omega_{a}, R_{a}\right)_{0}=\mathrm{H}^{0}\left(\mathscr{O}_{\mathbf{P}^{1}}(2)\right)=S_{2}(V),
$$

the second symmetric power of $V$. Making the corresponding identifications for $D_{b}$ with $\mathbf{P}^{1}=\mathbf{P}(W)$ for another 2-dimensional vectorspace $W$, we get

$$
\operatorname{Hom}_{R_{J}}\left(\omega_{J}, R_{J}\right)_{0}=S_{2}(V) \otimes_{k} S_{2}(W) .
$$

Since $\omega_{J}$ is a torsion free $R_{J}$-module of rank 1 , every nonzero map is a monomorphism. We set

$$
X_{\chi}=\operatorname{Proj} R_{J} / \chi\left(\omega_{J}\right)
$$

Since $R_{J} / \chi\left(\omega_{J}\right)$ is a 3-dimensional Gorenstein ring with trivial canonical divisor, $X_{\chi}$ may be regarded as a $\mathrm{K} 3$ surface, and its hyperplane sections will be canonically embedded curves.

As we have already remarked, the reduced structure on a $\mathrm{K} 3$ carpet is a rational normal scroll. In the terms above, such a scroll is determined by making an identification of $D_{a}$ with $D_{b}$, that is, by identifying $V$ with $W$. We may regard such an identification as an element of $\operatorname{Hom}_{k}(V, W)$, or, using the identification of $V$ and $V^{*}$ that we may make because $V$ is 2-dimensional, with an element of $V \otimes_{k} W$. Squaring this, we get an element of $S_{2}(V) \otimes$ $S_{2}(W)$ corresponding to the carpet $X$. (Note that if we were not working in characteristic 0 , some further care would be necessary!) Thus we see that the $\mathrm{K} 3$ carpets are quite special elements of the family $X_{\chi}$. It is not too hard to show that the general element may be described as follows: we may associate 
to $\chi$ a divisor $E_{\chi}$ of type $(2,2)$ on $\mathbf{P}^{1} \times \mathbf{P}^{1}=C_{a} \times C_{b}$, or equivalently as a correspondence of type $(2,2)$ from $C_{a}$ to $C_{b}$; generally, $E_{\chi}$ will be an elliptic curve, but it may degenerate to twice a conic, or become reducible. The union of the lines in $\mathbf{P}^{g}$ joining corresponding points is $X_{\chi}$. A hyperplane $H$ in $\mathbf{P}^{g}$ cuts $C_{a}$ in $a$ points and $C_{b}$ in $b$ points. Thus it determines on $E_{\chi}$ a set of $a$ pairs of points and a set of $b$ pairs of points. The hyperplane section $H \cap X_{\chi}$ is an embedding of $E_{\chi}$ with these $a+b$ pairs of points identified.

It is easy to write down a minimal free resolution of $R_{J}$ over the polynomial ring $T=T_{a} \otimes T_{b}$, the homogeneous coordinate ring of $\mathbf{P}^{g}$ : it is obtained by tensoring together (over $k$ ) a minimal free resolution of $R_{a}$ over $T_{a}$ and a minimal free resolution of $R_{b}$ over $T_{b}$. These resolutions may be written, equivariantly for the action of $G L(V)$ and $G L(W)$, as Eagon-Northcott complexes. Further, the minimal resolution of $\omega_{J}$ is the dual of the minimal resolution of $R_{J}$. A chosen element $\chi$ of $S_{2}(V) \otimes S_{2}(W)$, regarded as a map $\omega_{J} \rightarrow R_{J}$, lifts (uniquely up to homotopy) to a map $\delta_{\chi}$ of these resolutions. The mapping cylinder of $\delta_{\chi}$ is a resolution of the homogeneous coordinate ring $R_{X_{x}}$ of the K3 surface.

To obtain from this construction the graded betti numbers in a minimal resolution of $R_{X_{x}}$, it suffices to find the rank of the degree 0 (in the sense of the grading from $T$ ) part of the map $\delta_{\chi}$ of complexes. Because the EagonNorthcott complexes and their tensor product are minimal, the degree 0 part of $\delta_{\chi}$ is actually unique, and will thus be $G L(V) \times G L(W)$-equivariantly defined from $\chi$. We now make this explicit.

Anticanonical divisors on the rational normal curve. We first recall the resolution of $R_{a}$ over $T_{a}$. To simplify the notation, write $S_{i}$ for the free $T_{a}$-module $S_{i}(V) \otimes_{k} T_{a}$. The resolution $\mathscr{F}_{a}$ has the form:

$$
\begin{aligned}
T_{a} \stackrel{\phi_{1}}{\longleftarrow} \wedge^{2} S_{a-1}(-2) \leftarrow \ldots \stackrel{\phi_{m}}{\longleftarrow} \wedge^{m+1} \dot{S}_{a-1} \otimes S_{m-1}(-m-1) \\
\stackrel{\phi_{m+1}}{\longleftarrow} \ldots \stackrel{\phi_{a-1}}{\longleftarrow} \wedge^{a} S_{a-1} \otimes S_{a-2}(-a) \leftarrow 0 .
\end{aligned}
$$

The resolution of $\omega_{a}$ is the dual complex $\mathscr{F}^{*}(-a-1)$, which may conveniently be rewritten, using the isomorphisms $\left(\wedge^{m} S_{a-1}\right)^{*} \cong \wedge^{a-m} S_{a-1}$ given by a choice of a free generator for $\left(\wedge^{a} S_{a-1}\right)^{*}$, as

$$
\begin{aligned}
S_{a-2}(-1) \stackrel{\psi_{1}}{\longleftarrow} \wedge^{1} S_{a-1} \otimes S_{a-3}(-2) \ldots \stackrel{\psi_{m}}{\longleftarrow} \wedge^{m} S_{a-1} \otimes S_{a-2-m}(-m-1) \\
\stackrel{\psi_{m+1}}{\longleftarrow} \ldots \stackrel{\psi_{a-2}}{\longleftarrow} \wedge^{a-2} S_{a-1} \otimes S_{1}(-a+1) \stackrel{\psi_{a-1}}{\longleftarrow} T_{a}(-a-1) \leftarrow 0 .
\end{aligned}
$$

The map $\omega_{a} \rightarrow R_{a}$ corresponding to an element $x \in S_{2}(V)$ is given on generators by

$$
S_{a-2}(V) \stackrel{\delta_{0}(x)}{\longrightarrow}\left(T_{a}\right)_{1}=S_{a}(V), \quad e \mapsto x e,
$$

and lifts to a map $\delta(x): \mathscr{F}^{*}(-a-1) \rightarrow \mathscr{F}$ given by the formulas

$$
\begin{aligned}
\wedge^{m} S_{a-1} \otimes S_{a-2-m}(-m-1) & \stackrel{\delta_{m}(x)}{\longrightarrow} \wedge^{m+1} S_{a-1} \otimes S_{m-1}(-m-1), \\
e \otimes f & \mapsto \sum_{i} e \wedge\left(x u_{i} f\right) \otimes u_{i}^{\prime}
\end{aligned}
$$


for $1 \leq m \leq a-2$, where for some basis $v_{1}, v_{2} \in V$ the elements $u_{i}$ and $u_{i}^{\prime}$ are defined by the condition that

is the "trace" element

$$
\sum_{i} u_{i} \otimes u_{i}^{\prime} \in S_{m-1}(V) \otimes S_{m-1}(V)
$$

$$
\left(v_{1} \otimes v_{2}-v_{2} \otimes v_{1}\right)^{m-1} .
$$

The key point of the proof that the $\delta_{i}(x)$ commute with the $\phi_{i}$ and $\psi_{i}$ is the formula

$$
\sum_{i, j} \epsilon_{j} e_{i} \otimes \epsilon_{j}^{\prime *}\left(e_{i}\right)=0,
$$

where $\sum_{j} \epsilon_{j} \otimes \epsilon_{j}^{\prime *} \in V \otimes V^{*}$ is the usual trace element, and the action $\epsilon_{j}^{\prime *}\left(e_{i}\right)$ of $V^{*}$ on $S_{m-1}(V)$ is by derivations. We omit the details.

We can get a better idea of the nature of the maps $\delta(x)$ as follows. The mapping cylinder of $\delta(x)$ is a (nonminimal) resolution of an anticanonical divisor $A=A(x)$ on the rational normal curve $C_{a}$. Now an anticanonical divisor $A$ on $C_{a}$ is simply a divisor of degree 3 - that is, a scheme of three points. Of course $A$ spans a 2-plane. The minimal free resolution of $\mathscr{O}_{A}$ in $\mathbf{P}^{a}$ is the tensor product of the resolution of $A$ in the plane and the resolution of the plane itself, which is a Koszul complex. Note that for $1 \leq m \leq a-3$ the maps $\delta_{m}(x)$ are matrices of scalars. Their kernels and cokernels must add up to the minimal free resolution of $\mathscr{O}_{a}$. Comparing these sequences we deduce a self-dual family of natural exact sequences

$$
\begin{aligned}
& 0 \rightarrow \wedge^{m+1} S_{a-2} \rightarrow \wedge^{m} S_{a-1} \otimes S_{a-2-m} \stackrel{\delta_{m}(x)}{\longrightarrow} \\
& \wedge^{m+1} S_{a-1} \otimes S_{m-1} \rightarrow \wedge^{m-1} S_{a-2} \rightarrow 0 .
\end{aligned}
$$

associated with $x \in S_{2} V$. (To prove this, first compute the kernel of $\delta_{m}(x)$ by comparing the minimal resolution and the mapping cylinder, as above; then use the fact that $\delta_{m}(x)$ is isomorphic to the dual of the map $\delta_{a-1-m}(x)$.)

As suggested by the notation, we may regard $\delta_{m}(x)$ as a family of maps of free modules defined over the polynomial ring in 3 variables $Q:=k\left[S_{2}(V)\right]$, and in these terms the exact sequences above become complexes

$$
\begin{aligned}
0 \rightarrow \wedge^{m+1} S_{a-2}(-m) \rightarrow & \wedge^{m} S_{a-1} \otimes S_{a-2-m} \stackrel{\delta_{m}}{\longrightarrow} \\
& \wedge^{m+1} S_{a-1} \otimes S_{m-1}(1) \rightarrow \wedge^{m-1} S_{a-2}(a-m) .
\end{aligned}
$$

over $Q$. For example, if we take $m=1$, we get the resolution of the $(a-1-m)$ th power of the maximal ideal of $Q$, written in a peculiar way. It would be interesting to understand these complexes in general.

Anticanonical divisors on $J$. The minimal free resolution of $R_{J}$ over $T$ is

$$
\mathscr{F}:=\mathscr{F}_{a} \otimes_{k} \mathscr{F}_{b} \text {, }
$$

and the minimal free resolution of $\omega_{J}$ is

$$
\mathscr{G}:=\mathscr{F}_{a}^{*} \otimes_{k} \mathscr{F}_{b}^{*}(-g-1) .
$$

Given an element $\chi:=\sum x_{i} \otimes y_{i} \in S_{2}(V) \otimes S_{2}(W)$, we get a (nonminimal) resolution of the homogeneous coordinate ring of the corresponding anticanonical divisor $X_{\chi}$ by taking the mapping cylinder of the map of complexes

$$
\delta_{\chi}=\sum_{i, j} \delta\left(x_{i}\right) \otimes \delta\left(y_{j}\right): \mathscr{G} \rightarrow \mathscr{F} .
$$


The canonical ribbon conjecture, which says that the minimal free resolution of $X_{\chi}$ has no $(a-1)$ st syzygies of degree $a+1$ when $\chi$ is a square of an element of rank 2 is thus equivalent to the statement that for generic $\chi$ the map

$$
\delta_{\chi, a-1}:\left(\mathscr{G}_{a-1}\right)_{a+1} \rightarrow\left(\mathscr{F}_{a-1}\right)_{a+1}
$$

is surjective. Of course proving the surjectivity for general would prove the analogue of Green's conjecture for some other degenerate K3 surface of Clifford index $a$, which would be just as interesting.

Writing out the terms in question, one must show that for all $m, n$ with $m \geq 1, n \geq 1, m+n=a-1<b$ the map

$$
\begin{aligned}
\sum_{i, j} \delta_{m}\left(x_{i}\right) \otimes \delta_{n}\left(y_{j}\right): \wedge^{m} S_{a-1} \otimes S_{a-2-m} & \otimes \wedge^{n} S_{b-1} \otimes S_{b-2-n} \\
\wedge^{m+1} S_{a-1} \otimes S_{m-1} & \otimes \wedge^{n+1} S_{b-1} \otimes S_{n-1}
\end{aligned}
$$

is surjective for suitable $x_{i}$ and $y_{j}$.

The star construction. We can abstract the construction above as follows: Since we are interested in these things for generic values of the $x_{i}$ and $y_{j}$ we might as well take these as variables. The maps $\delta_{m}$ and $\delta_{n}$ used above are then defined over the polynomial rings $Q=k\left[x_{1}, x_{2}, x_{3}\right]$ and $Q^{\prime}=k\left[y_{1}, y_{2}, y_{3}\right]$ respectively.

Suppose, in general, that we are given a map $d: F \rightarrow G$ of free modules over $Q=k\left[x_{1}, \ldots, x_{s}\right]$ and another map $d^{\prime}: F^{\prime} \rightarrow G^{\prime}$ of free modules over $Q^{\prime}=k\left[y_{1}, \ldots, y_{t}\right]$. Suppose further that, as in the case above, each of these maps is represented by a matrix of linear forms. We define a map

$$
d * d^{\prime}: F \otimes_{k} F^{\prime} \rightarrow G \otimes_{k} G^{\prime},
$$

the "star product" of $d$ and $d^{\prime}$, over the ring $\tilde{Q}:=k\left[z_{i, j}\right]_{1 \leq i \leq s, 1 \leq j \leq t}$, by taking $d \otimes_{k} d^{\prime}$ over the ring $Q \otimes_{k} Q^{\prime}$, and then replacing the product $x_{i} y_{j}$ by the variable $z_{i, j}$. This is legitimate because the natural map $\tilde{Q} \rightarrow Q \otimes_{k} Q^{\prime}$ maps the linear forms of $\tilde{Q}$ isomorphically to the bilinear forms of $Q \otimes_{k} Q^{\prime}$. To show that the maps above are surjective for generic choice of $x_{i}$ and $y_{j}$ is to show that are the *-products of the "easy" maps $\delta_{m}$ and $\delta_{n}$ have maximal rank over $\tilde{Q}$.

It may clarify matters to give a simple example. If we are to have a nontrivial computation then we must have $m \geq 1, n \geq 1, m+n=a-1$ and $a \leq b, g=$ $a+b+1$. The first case in which this is possible is $a=b=3, g=7$. Here we must take $m=n=1$. In this case $d_{2}$ is just the middle map of the koszul complex in 3 variables

$$
\delta_{2}\left(x_{1} v_{1}^{2}+x_{2} v_{1} v_{2}+x_{3} v_{2}^{2}\right)=\left(\begin{array}{ccc}
0 & v_{3} & -v_{2} \\
-v_{3} & 0 & v_{1} \\
v_{2} & -v_{1} & 0
\end{array}\right) .
$$

Of course these maps have rank only 2 ; they are not themselves of maximal 
rank since they are skew symmetric and of odd size. However, the star product $\delta_{2}\left(x_{1} v_{1}^{2}+x_{2} v_{1} v_{2}+x_{3} v_{2}^{2}\right) * \delta_{2}\left(y_{1} v_{1}^{2}+y_{2} v_{1} v_{2}+y_{3} v_{2}^{2}\right)=$

$$
\left(\begin{array}{ccccccccc}
0 & 0 & 0 & 0 & z_{3,3} & -z_{3,2} & 0 & -z_{2,3} & z_{2,2} \\
0 & 0 & 0 & -z_{3,3} & 0 & z_{3,1} & z_{2,3} & 0 & -z_{2,1} \\
0 & 0 & 0 & z_{3,2} & -z_{3,1} & 0 & -z_{2,2} & z_{1,1} & 0 \\
0 & -z_{3,3} & z_{3,2} & 0 & 0 & 0 & 0 & z_{1,3} & -z_{1,2} \\
z_{3,3} & 0 & -z_{3,1} & 0 & 0 & 0 & -z_{1,3} & 0 & z_{1,1} \\
-z_{3,2} & z_{3,1} & 0 & 0 & 0 & 0 & z_{1,2} & -z_{1,1} & 0 \\
0 & z_{2,3} & -z_{2,2} & 0 & -z_{1,3} & z_{1,2} & 0 & 0 & 0 \\
-z_{2,3} & 0 & z_{2,1} & z_{1,3} & 0 & -z_{1,1} & 0 & 0 & 0 \\
z_{2,2} & -z_{2,1} & 0 & -z_{1,2} & z_{1,1} & 0 & 0 & 0 & 0
\end{array}\right)
$$

is of maximal rank as long as the characteristic of $k$ is not 2; it is a symmetric $9 \times 9$ matrix with zeros on the diagonal, so in characteristic 2 it can have rank only 8 . The exception for characteristic 2 corresponds precisely to Schreyer's observation that the general canonical curve of genus 7 does not in fact satisfy Green's conjecture.

Numerical evidence. We finish by exhibiting the betti numbers for canonical ribbons of Clifford index $\geq 2$ and genus $\leq 12$, as computed by the program Macaulay [1990], in characteristic 31,991. We give only the "2-linear" part. The rest may be reproduced by using the symmetry of the resolution. Thus a listing

$$
\begin{array}{ccccc}
\text { genus } & \text { index } \\
7 & 2 & 10 & 16 & 9
\end{array}
$$

is to be read as the assertion that a rational ribbon of genus 7 and Clifford index 2 has a minimal free resolution of the form

$$
\begin{aligned}
\mathscr{O} \leftarrow \mathscr{O}(-2)^{10} \leftarrow \mathscr{O}(-3)^{16} \oplus \mathscr{O}(-4)^{9} & \leftarrow \mathscr{O}(-4)^{9} \oplus \mathscr{O}(-5)^{16} \\
& \leftarrow \mathscr{O}(-6)^{10} \leftarrow \mathscr{O}(-8) \leftarrow 0,
\end{aligned}
$$

where $\mathscr{O}$ denotes $\mathscr{O}_{\mathbf{P}^{8-1}}(a)=\mathscr{O}_{\mathrm{P}^{6}}(a)$. In the notation used by the "betti" operation of the program Macaulay this would be written

$$
\begin{array}{llllll}
1 & - & - & - & - & - \\
- & 10 & 16 & 9 & - & - \\
- & - & 9 & 16 & 10 & -
\end{array}
$$

The following betti numbers were computed by Macaulay:

$\begin{array}{ccrrrrrrrrrr}\text { genus } & \text { index } & & & & & & & & \\ 5 & 2 & 3 & & & & & & & \\ 6 & 2 & 6 & 5 & & & & & & \\ 7 & 2 & 10 & 16 & 9 & & & & & \\ 8 & 2 & 15 & 35 & 35 & 14 & & & & \\ 9 & 2 & 21 & 64 & 90 & 64 & 20 & & & \\ 10 & 2 & 28 & 105 & 189 & 189 & 105 & 27 & & \\ 11 & 2 & 36 & 160 & 350 & 448 & 350 & 160 & 35 & \\ 12 & 2 & 45 & 231 & 594 & 924 & 924 & 594 & 231 & 44\end{array}$




$\begin{array}{ccccccccc}\begin{array}{c}\text { genus } \\ 7\end{array} & \text { index } & & & & & & & \\ 8 & 3 & 10 & 16 & & & & & \\ 9 & 3 & 15 & 35 & 21 & & & & \\ 10 & 3 & 21 & 64 & 70 & 24 & & & \\ 11 & 3 & 36 & 160 & 315 & 336 & 210 & 48 & \\ 12 & 3 & 45 & 231 & 550 & 756 & 672 & 342 & 63 \\ \text { genus } & \text { index } & & & & & & & \\ 9 & 4 & 21 & 64 & 70 & & & & \\ 10 & 4 & 28 & 105 & 162 & 84 & & & \\ 11 & 4 & 36 & 160 & 315 & 288 & 100 & & \\ 12 & 4 & 45 & 231 & 550 & 693 & 455 & 125 \\ \text { genus } & \text { index } & & & & & & & \\ 11 & 5 & 36 & 160 & 315 & 288 & & & \\ 12 & 5 & 45 & 231 & 550 & 693 & 330 & \end{array}$

These numbers support the Canonical Ribbon Conjecture stated at the beginning of this paper.

It is interesting to compare these betti numbers with those computed by Schreyer [1986] for smooth curves of Clifford index $\geq 2$, genus $\leq 8$, over a field of characteristic 0 . For curves of Clifford index 2 and genus 7 or 8 , the betti numbers of the smooth curves agree with the corresponding betti numbers of the ribbons if the curve has a $g_{6}^{2}$. In the case of a smooth curve of Clifford index 2 with no $g_{6}^{2}$, the betti numbers computed by Schreyer are instead:

$\begin{array}{cccccc}\text { genus } & \text { index } & & & & \\ 7 & 2 & 10 & 16 & 3 & \\ 8 & 2 & 15 & 35 & 25 & 4\end{array}$

\section{APPENDIX: OSCULATING BUNDLES OF THE RATIONAL NORMAL CURVE}

We assume for simplicity that the ground field $k$ has characteristic 0 , although our results could be reformulated for the case of arbitrary $k$.

The following results identify the quotients of any two osculating bundles of the rational normal curve in terms of the representation theory of $S L(2)$. Though special cases, at least, are well known, we do not know a convenient reference. For simplicity, we work over a field $k$ of characteristic 0 , although the second of the two proofs we give for the main result may be adapted to work in any characteristic.

For each $r$, let $\mathbf{P}^{1} \cong D \subset \mathbf{P}^{r}$ be the rational normal curve, and let $T_{r}=\left.T_{\mathbf{P}^{r}}\right|_{\mathbf{P}^{1}}$ be the restricted tangent bundle. There is an obvious embedding $T_{\mathbf{P}^{1}}=T_{1} \subset T_{r}$, and in fact the $T_{m}$ form a flag of bundles

$$
0 \subset T_{1} \subset T_{2} \subset \cdots \subset T_{r} \ldots
$$

In terms of the embedding in a given $\mathbf{P}^{r}$, this flag is realized geometrically as the flag of osculating bundles of $D$ :

Proposition 5A.1. The subbundle of $T_{r}$ whose fiber at a point $p \in D$ is the set of tangent vectors lying in the osculating $m$-plane to $D$ at $p$ is isomorphic to 
$T_{m}$. Further, the inclusions $T_{m} \subset T_{n}$ obtained in this way are independent of $r$.

Proof. For the proof we shall need a more formal description of the subbundle of $T_{r}$ whose fibers are the osculating $m$-planes: we shall temporarily call it $\mathrm{Osc}_{m}$.

If we write $L$ for the line bundle $\mathscr{O}_{\mathbf{P}^{1}}(r)$ on $D$, and let $\mathscr{P}^{m}(L)$ be the bundle of principle parts of $L$ of order $m$, then there is a natural map $\mathrm{H}^{0}(L) \otimes \mathscr{O}_{D} \rightarrow$ $\mathscr{P}^{m}(L)$ that, locally at each point, takes a section to its Taylor series. Let $\mathscr{E}_{m}$ be the image of the dual map, twisted by $L$. The bundle that we have called $O s c_{m}$ is the image of $\mathscr{E}_{m}$ under the natural map $\left.\mathrm{H}^{0}(L)^{*} \otimes L \rightarrow T_{\mathbf{P r}}\right|_{D}$; that is, there is a commutative diagram

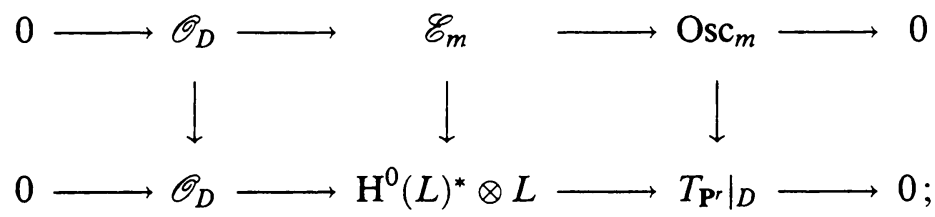

see Piene [1977] for details.

We shall show that the osculating bundles and inclusions are the same in $\mathbf{P}^{r}$ and $\mathbf{P}^{r-1}$ by projecting from a point $p$ of the rational normal curve $D$. In particular, $T_{r-1}=\mathrm{Osc}_{r-1}$. By induction this proves the theorem.

Write $L=\mathscr{O}_{\mathbf{P}^{1}}(r)$ for the embedding line bundle. The natural inclusion $L(-p) \subset L$ induces diagrams

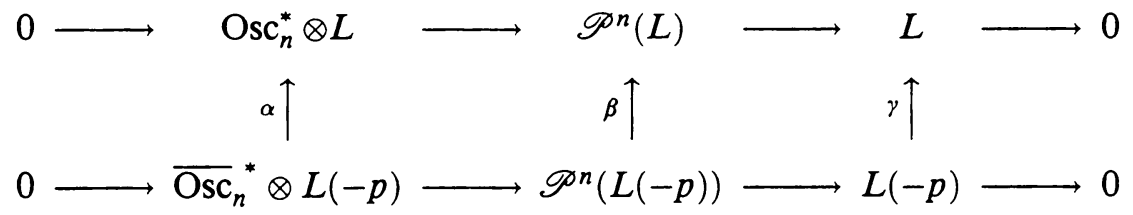

where we have written $\overline{\mathrm{Osc}}_{n}$ for the osculating bundle of the rational normal curve in $\mathbf{P}^{r-1}$. To show that $\overline{\mathrm{Osc}}_{n} \cong \mathrm{Osc}_{n}$ it suffices to show that the map labelled $\alpha$ in the diagram is isomorphic to the inclusion of $\operatorname{Osc}_{n}^{*} \otimes L(-p)$ in $\operatorname{Osc}_{n}^{*} \otimes L$, or equivalently that the cokernel of $\alpha$ is the the sheaf $k(p)^{n}$. Since the inclusions of one osculating bundle in another are compatible with these commutative diagrams, this will suffice to show that the inclusions are also independent of $r$.

Since $\beta$ and $\gamma$ are isomorphisms away from $p$, they are inclusions of sheaves, and it follows that the same is true for $\alpha$. Since the cokernel of $\gamma$ is obviously $k(p)$, it suffices to show that the cokernel of $\beta$ is $k(p)^{n+1}$. Since locally $\mathscr{P}^{n}(L)$ looks like $\sum_{0}^{n} L \otimes d t^{i}$ this is clear.

To describe the quotients $T_{m} / T_{n}$ equivariantly we need a notation for the representations of $\operatorname{SL}(2)$. If $V$ is a 2-dimensional vectorspace, $S:=\operatorname{Sym}(V)$ is the symmetric algebra, and we identify the rational normal curve as $D=\operatorname{Proj} S$, then $\operatorname{SL}(2)=\operatorname{SL}(V)$ acts naturally on $\mathrm{D}$ and on

$$
S_{n}:=\operatorname{Sym}_{n}(V)=\mathrm{H}^{0}\left(\mathscr{O}_{\mathbf{P}^{\mathbf{1}}}(n)\right),
$$

and these are the irreducible representations of $\operatorname{SL}(V)$. 
Proposition 5A.2. With notation as above,

$$
T_{m} / T_{n} \cong S_{m-n-1} \otimes \mathscr{O}_{\mathbf{P}^{1}}(m+n+1)
$$

equivariantly for the action of $\mathrm{SL}(2)$.

We give two proofs - the first, which was suggested by Joe Harris, is appealingly geometric. The second, by free resolutions, gives slightly more information, and is essentially characteristic free.

Geometric Proof. First we compute chern classes: from the exact sequences

$$
0 \rightarrow \Omega_{D}^{n} \otimes L \rightarrow \mathscr{P}^{n}(L) \rightarrow \mathscr{P}^{n-1}(L) \rightarrow 0
$$

and the "initial case"

$$
\mathscr{P}^{0}(L)=L
$$

we easily derive

from which we get

$$
c_{1}\left(\mathscr{P}^{n}(L)\right)=(n+1)(r-n),
$$

$$
c_{1}\left(T_{m} / T_{n}\right)=(m-n)(m+n+1) .
$$

Next we shall show that $T_{m} / T_{n}$ is a direct sum of equal line bundles so that, for some $S L(2)$ representation $U$, we have

$$
T_{m} / T_{n} \cong U \otimes \mathscr{O}_{\mathbf{P}^{1}}(m+n+1) .
$$

From the construction, it will appear that these line bundles can be chosen from a single family parametrized by a 1-dimensional projective $\mathrm{SL}(2)$ orbit. Since the only representations of SL(2) whose projectivization contains such a curve are the irreducible representations $S_{j}$, this will conclude the proof. (Here we are using the characteristic 0 hypothesis.)

It remains to produce the family of subbundles. The curve $\mathbf{P}^{1}=D \subset \mathbf{P}^{m}$ will itself form the parameter space: for each point $p \in D$ we define a line bundle $M[p] \subset T_{m}$ whose fiber at a point $x \in D$ other than $p$ is the line spanned by $x$ and $p$, regarded as a line in the tangent space to $\mathrm{P}^{m}$ at $x$ modulo the osculating $n$-plane to $D$ at $x$. This defines $M[p]$ as a bundle on $D-p$. There is of course a unique extension of $M[p]$ to a bundle on all of $D:$ its fiber at $p$ is the osculating $(n+1)$-plane at $p$ modulo the osculating $m$-plane.

We claim that for any set of $m-n$ distinct points $p_{1}, \ldots, p_{n-m}$ on $D$, we have

$$
T_{m} / T_{n}=\bigoplus_{1}^{m-n} M\left[p_{i}\right]
$$

This follows from the fact that any collection of osculating spaces to the rational normal curve is "as linearly independent as possible": in our case, if the line bundles in question failed to span at some point $x$, then the osculating $n$-space at $x$ together with the points $p_{i}$ (or the osculating $(n+1)$-space at $x$ together with all the $p_{i}$ if $x$ is one of the $p_{i}$ ) would be contained in a hyperplane, which would then meet $D$ at least $m+1$ times, contradicting the fact that the degree of $D$ is $m$.

Algebraic Proof. We shall actually make explicit the maps in the sequences defining the bundles $T_{m}$ and in the exact sequences

$$
0 \rightarrow T_{n} \rightarrow T_{m} \rightarrow S_{m-n-1} \otimes \mathscr{O}_{\mathbf{P}^{1}}(m+n+1) .
$$


All the maps of bundles with which we are concerned are of two types, which we shall first describe abstractly. Let

$$
\phi_{a, b}: S_{a} \rightarrow S_{a+b} \otimes S_{b}
$$

be the map of SL(2)-representations obtained by multiplying with the canonical "trace" element in $S_{b} \otimes S_{b}$. As an element of $S_{b} \otimes S_{b}$ this is $(s \otimes t-t \otimes s)^{b}$ where $s, t$ is a basis of $V=S_{1}$. If we write $\hat{s}, \hat{t} \in V^{*}$ for the basis dual to $s, t \in V$ then the usual trace element is $s \otimes \hat{s}+t \otimes \hat{t} \in V \otimes V^{*}$. Under the equivariant identification $V^{*} \cong \wedge^{2-1} V=V$, which sends

$$
\hat{s} \mapsto t, \quad \hat{t} \mapsto-s .
$$

This trace element goes to the element $s \otimes t-t \otimes s$, which is why we call it the trace as well.

This map induces an equivariant map of sheaves, for which we shall use the same name:

$$
\phi_{a, b}: S_{a} \otimes \mathscr{O}_{\mathscr{P}_{1}} \rightarrow S_{a+b} \otimes \mathscr{O}_{\mathbf{P}^{1}}(b) .
$$

Explicitly, if $i+j=a$ and we write $\left[s^{p} t^{q}\right]$ for the corresponding basis element of $S_{p+q} \otimes \mathscr{O}_{\mathbf{P} 1}$ then

$$
\phi_{a, b}:\left[s^{i} t^{j}\right] \mapsto \sum_{m+n=a+b}(-1)^{n-j}\left(\begin{array}{c}
b \\
m-i
\end{array}\right) s^{n-j} t^{m-i}\left[s^{m} t^{n}\right] .
$$

The second type of map that we shall need is closely related. Let

$$
\psi_{a, b}^{*}: D_{b}\left(V^{*}\right) \rightarrow D_{a+b}\left(V^{*}\right) \otimes S_{a},
$$

where $D_{b}\left(V^{*}\right)$ denotes the $b$ th graded component of the divided power algebra on $V^{*}$, be the map of $\mathrm{SL}(2)$-representations obtained by multiplying with the canonical "trace" element in $D_{a}\left(V^{*}\right) \otimes S_{a}$. In terms of the basis and dual basis for $V$ and $V^{*}$ introduced above, this element is the divided power

$$
(\hat{s} \otimes s+\hat{t} \otimes t)^{(a)}=\hat{s}^{(a)}+\hat{s}^{(a-1)} t+\hat{s}^{(a-2)} \hat{t}^{(2)}+\ldots
$$

This map induces an equivariant map of sheaves, for which we use the same name

$$
\psi_{a, b}^{*}: D_{b}\left(V^{*}\right) \otimes \mathscr{O}_{\mathbf{P}^{1}} \rightarrow D_{a+b}\left(V^{*}\right) \otimes \mathscr{O}_{\mathbf{P}^{1}}(a) .
$$

Dualizing, twisting with $\mathscr{O}_{\mathbf{P}^{1}}(a)$, and using the canonical isomorphism $D_{c}\left(V^{*}\right)^{*}$ $\cong S_{c}$ that makes $\left\{s^{m} t^{n}\right\}$ the dual basis to $\left\{\hat{s}^{m} \hat{t}^{n}\right\}$ we get the map we want,

$$
\psi_{a, b}: S_{a+b} \otimes \mathscr{O}_{\mathbf{P}^{1}} \rightarrow S_{b} \otimes \mathscr{O}_{\mathbf{P}^{1}}(a) .
$$

Explicitly, with notation as above: if $m+n=a+b$, then

$$
\psi_{a, b}:\left[s^{m} t^{n}\right] \mapsto \sum_{i+j=b}\left(\begin{array}{c}
m \\
i
\end{array}\right)\left(\begin{array}{c}
n \\
j
\end{array}\right) s^{m-i} t^{n-j}\left[s^{i} t^{j}\right] .
$$

We claim that (always in characteristic 0 ) these maps form, for every $a, b$, an exact sequence of sheaves:

$$
\begin{aligned}
E_{a, b}: 0 \rightarrow S_{a-1} \otimes \mathcal{O}_{\mathbf{P} 1} \stackrel{\phi_{a-1, b}}{\longrightarrow} & S_{a+b-1} \otimes \mathcal{O}_{\mathbf{P}}(b) \\
& \stackrel{\psi_{a+b-1, a}(b)}{\longrightarrow} S_{b-1} \otimes \mathscr{O}_{\mathbf{P}^{1}}(a+b) \rightarrow 0 .
\end{aligned}
$$


In fact, we claim that $E_{a, b} \otimes \mathscr{O}_{\mathbf{P}^{1}}(a+1)$ is isomorphic to the sequence

$$
0 \rightarrow T_{a} \rightarrow T_{a+b} \rightarrow T_{b} \rightarrow 0 .
$$

Further, the "Euler sequence" defining $T_{b}$,

$$
0 \rightarrow \mathscr{O}_{\mathbf{P}^{1}} \rightarrow S_{b}^{*} \otimes \mathscr{O}_{\mathbf{P}^{1}}(b) \rightarrow T_{b} \rightarrow 0,
$$

is isomorphic to the sequence $E_{1, b}$. Of course this will prove the proposition.

First, to prove that the sequences $E_{a, b}$ are exact, we appeal to the criterion of exactness of Buchsbaum-Eisenbud [1973]. It is easy to check directly the the $E_{a, b}$ are complexes, and from inspection the ideals of maximal minors of the two maps contain powers of both $s$ and $t$, so the conditions of the criterion are immediate.

Next, that $E_{1, b}$ is the Euler sequence is also clear, since the map

$$
\mathscr{O}_{\mathbf{P}^{1}} \rightarrow S_{b}^{*} \otimes \mathscr{O}_{\mathbf{P}^{1}}(b)
$$

in the Euler sequence is multiplication by the $b$ th power of the trace element.

Finally, to identify the sequence $(*)$ with $E_{a, b} \otimes \mathscr{O}_{\mathbf{P}^{1}}(a+1)$ it suffices to show that the diagram

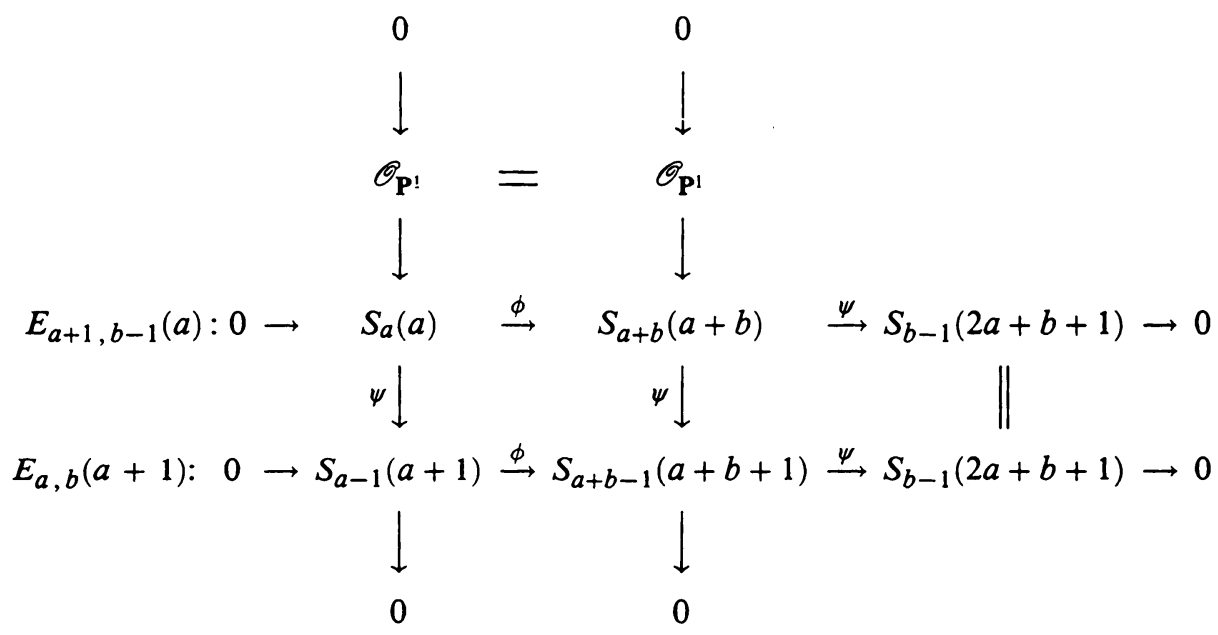

commutes, where the two long vertical columns are the Euler sequences, and we have written $S_{a}(a)$ for $S_{a} \otimes \mathscr{O}_{\mathbf{P}^{1}}(a)$, etc. Note that there is, up to scalar, only one inclusion of representations

$$
S_{a} \subset S_{a+b} \otimes S_{b}
$$

so the inclusion $T_{n} \subset T_{m}$ induced by the diagram must be the geometrically defined one.

Since all the maps have been given explicitly, this is presumably only an exercise. But it is possible to say that the maps must commute. To see this, note that we need only check the commutativity of the part involving the two Euler sequences, since the commutativity of the lower right-hand box (at least up to a scalar) is then forced by the irreducibility of $S_{m}$. Similarly, to check the commutativity of the part involving the Euler sequences (up to scalar), we 
need only check the commutativity of the upper left-hand box

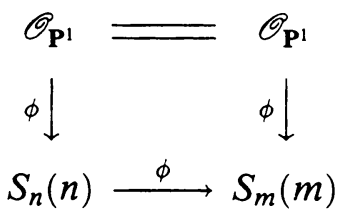

because $S_{n}$ is irreducible. This is easy, since the maps labelled $\phi$ are all given by multiplication by powers of the trace element.

\section{REFERENCES}

D. Bayer and M. Stillman, Macaulay: A system for computation in algebraic geometry and commutative algebra, Available from the authors or by anonymous $\mathrm{ftp}$ as follows. $\mathrm{ftp}$ zariski.harvard.edu, login: anonymous, Password: any, cd Macaulay, binary, get M3.tar, quit, tar xf M3.tar.

K. Chandler, Geometry of dots and ropes, Trans. Amer. Math. Soc. 347 (1995), 767-784.

D. A. Buchsbaum and D. Eisenbud, What makes a complex exact?, J. Algebra 25 (1973), 259-268.

D. Eisenbud, Green's conjecture; an orientation for algebraists, Sundance 91: Proceedings of a Conference on Free Resolutions in Commutative Algebra and Algebraic Geometry, Jones and Bartlett, 1992, pp. 51-78.

D. Eisenbud and M. L. Green, Clifford indices of ribbons, Trans. Amer. Math. Soc. 347 (1995), 757-765.

D. Eisenbud and J. Harris, Finite projective schemes in linearly general position, J. Algebra Geom. 46 (1992), 15-30.

, On varieties of minimal degree, Algebraic Geometry; Bowdoin 1985. Proc. Sympos. Pure Math., vol. 46, part 1, Amer. Math. Soc., Providence, RI, 1987, pp. 3-14.

L.-Y. Fong, Rational ribbons and deformation of hyperelliptic curves, J. Algebraic Geom. 2 (1993), 295-307.

L. Gruson and C. Peskine, Courbes dans l'espace projectif, varietés de sécantes, Enumerative Geometry and Projective Geometry, Progress in Math., vol. 24, (P. Le Barz and Y. Hervier, eds.), Birkhäuser, Boston, MA, 1982, pp. 1-31.

R. Hartshorne, Curves with high self intersection on algebraic surfaces, Publ. Math. IHES 36 (1969), 111-125.

K. Hulek and A. Van de Ven, The Horrocks-Mumford bundle and the Ferrand construction, Manuscripta Math. 50 (1985), 313-335.

S. Lichtenbaum and M. Schlessinger, The cotangent complex of a morphism, Trans. Amer. Math. Soc. 128 (1967), 41-70.

R. Piene, Numerical characters of a curve in projective -space, Real and Complex Singularities, Oslo 1976, (P. Holm, ed.), Sijthoff and Noordhoff, The Netherlands, 1977, pp. 475-498.

F. O. Schreyer, Syzygies of canonical curves and special linear series, Math. Ann. 275 (1986), 105-137.

E. Sernesi, Topics on families of projective schemes, Queen's Papers in Pure and Appl. Math. 73, Queen's Univ., Kingston, Canada, 1986.

Department of Mathematics, Barnard College, Columbia University, New York, New YORK 10027

E-mail address: dab@math.columbia.edu

Department of Mathematics, Brandeis University, Waltham Massachusetts 02254

E-mail address: eisenbud@math.brandeis.edu 